\title{
Boundary-Layer Instability Measurements in a Mach-6 Quiet Tunnel
}

\author{
Dennis C. Berridge* \\ NASA Langley Research Center, Hampton, VA 23681 \\ School of Aeronautics and Astronautics, Purdue University, West Lafayette, IN 47907-1282 \\ Christopher A.C. Ward ${ }^{\dagger}$ Ryan P.K. Luersen`, Amanda Chou $†$ \\ Andrew D. Abney $\dagger^{\dagger}$ and Steven P. Schneider ${ }^{\ddagger}$ \\ School of Aeronautics and Astronautics, Purdue University, West Lafayette, IN 47907-1282
}

Several experiments have been performed in the Boeing/AFOSR Mach-6 Quiet Tunnel at Purdue University. A $7^{\circ}$ half angle cone at $6^{\circ}$ angle of attack with temperature-sensitive paint (TSP) and PCB pressure transducers was tested under quiet flow. The stationary crossflow vortices appear to break down to turbulence near the lee ray for sufficiently high Reynolds numbers. Attempts to use roughness elements to control the spacing of hot streaks on a flared cone in quiet flow did not succeed. Roughness was observed to damp the second-mode waves in areas influenced by the roughness, and wide roughness spacing allowed hot streaks to form between the roughness elements. A forward-facing cavity was used for proof-of-concept studies for a laser perturber. The lowest density at which the freestream laser perturbations could be detected was $1.07 \times 10^{-2} \mathrm{~kg} / \mathrm{m}^{3}$. Experiments were conducted to determine the transition characteristics of a streamwise corner flow at hypersonic velocities. Quiet flow resulted in a delayed onset of hot streak spreading. Under low Reynolds number flow hot streak spreading did not occur along the model. A new shock tube has been built at Purdue. The shock tube is designed to create weak shocks suitable for calibrating sensors, particularly PCB-132 sensors. PCB-132 measurements in another shock tube show the shock response and a linear calibration over a moderate pressure range.

\section{Nomenclature}

$a_{0} \quad$ speed of sound based on stagnation temperature

$D \quad$ cavity diameter

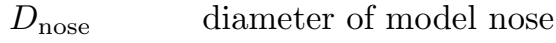

$\delta \quad$ average bow shock standoff distance

$E_{\text {pulse }} \quad$ pulse energy of laser going into perturber optics

$f_{1 n} \quad$ theoretical natural resonant frequency of cavity

$f_{\text {eff }} \quad$ effective focal length of multi-element lens

$k \quad$ thermal conductivity

$L \quad$ cavity depth

$L *=L+\delta$ distance between cavity base and average location of bow shock

$M \quad$ Mach number

$p \quad$ pressure

$\dot{q} \quad$ heat flux

$\rho \quad$ density

$t \quad$ time after tunnel start

\footnotetext{
${ }^{*}$ Research Assistant and Co-op Student, Aerothermodynamics Branch, Student Member, AIAA

$\dagger$ Research Assistant, Student Member, AIAA

${ }^{\ddagger}$ Professor, Associate Fellow, AIAA
} 
T temperature

$z \quad$ axial tunnel coordinate, $z=0$ at BAM6QT throat, increases with downstream distance

$z_{\text {nose }} \quad$ axial location of model nose

$\omega_{1 n} \quad$ theoretical natural resonant frequency of cavity

Subscripts

$0, \mathrm{i} \quad$ initial stagnation conditions

0,1 freestream stagnation conditions (upstream of the bow shock)

$0,2 \quad$ stagnation conditions downstream of the bow shock.

$\infty \quad$ in freestream

Superscripts

, RMS fluctuations

\section{Introduction}

Laminar to turbulent transition in hypersonic boundary layers is important for prediction and control of heat transfer, skin friction, and other boundary layer properties. Vehicles that spend extended periods at hypersonic speeds may be critically affected by the uncertainties in transition prediction, depending on their Reynolds numbers. However, the mechanisms leading to transition are still poorly understood, even in low-noise environments.

Many transition experiments have been carried out in conventional ground-testing facilities over the past 50 years. $^{1}$ However, these experiments are contaminated by the high levels of noise that radiate from the turbulent boundary layers normally present on the wind tunnel walls. ${ }^{2}$ These noise levels, typically $0.5-1 \%$ of the mean, are an order of magnitude larger than those observed in flight. ${ }^{3,4}$ These high noise levels can cause transition to occur an order of magnitude earlier than in flight. ${ }^{2,4}$ In addition, the mechanisms of transition operational in small-disturbance environments can be changed or bypassed altogether in high-noise environments; these changes in the mechanisms can change the parametric trends in transition. ${ }^{3}$ Mechanismbased prediction methods must be developed, supported in part with measurements of the mechanisms in quiet wind tunnels.

\section{A. The Boeing/AFOSR Mach-6 Quiet Tunnel}

Quiet tunnels have much lower noise levels than conventional wind tunnels. The freestream noise level of a quiet tunnel is about $0.1 \%$ or less. ${ }^{2}$ The use of such tunnels provides insight into transition in a quiet, flight-like condition. Purdue University's Boeing/AFOSR Mach-6 Quiet Tunnel (BAM6QT) is one of two operational hypersonic quiet tunnels, as NASA Langley's former Mach-6 quiet tunnel is now operational at Texas A\&M. ${ }^{5}$

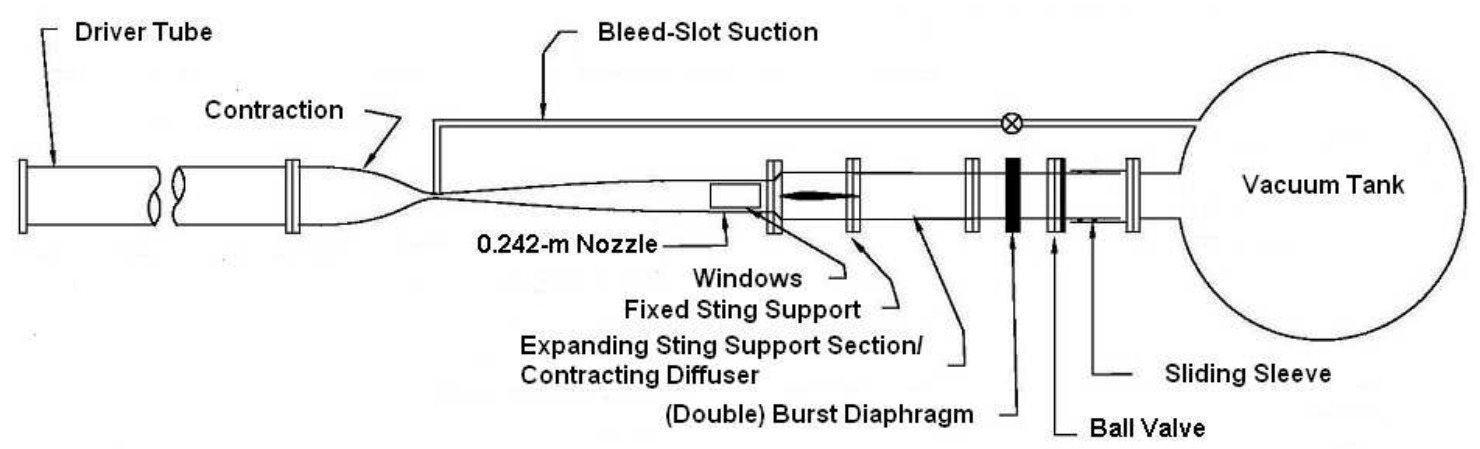

Figure 1. The Boeing/AFOSR Mach-6 Quiet Tunnel

Purdue's BAM6QT was designed as a Ludwieg tube in order to reduce the cost of running the tunnel. The 
Ludwieg tube involves a long pipe, a converging-diverging nozzle, test section, diffuser, burst diaphragms, and a vacuum tank (Figure 1). The tunnel is run by pressurizing the driver tube to a desired stagnation pressure and pumping the downstream portion to vacuum. Bursting the diaphragms starts the flow, which sends a shock wave downstream into the vacuum tank and an expansion wave upstream. The expansion wave reflects between the contraction and the upstream end of the driver tube throughout the length of the run, causing the stagnation pressure to drop quasi-statically in a stair-step fashion. In the BAM6QT's quiet configuration, air is bled from the throat of the nozzle using a fast valve, allowing a new boundary layer to grow on the divergent portion of the nozzle wall. To run the tunnel in a conventional configuration, the bleed valves are closed. The extended length and high polish of the nozzle allows the boundary layer to remain laminar to fairly high stagnation pressures. Visual access is provided by thick acrylic windows. The windows are exposed to full stagnation pressure before the run, so the structural strength of the windows is a concern. At lower pressures, a large acrylic insert can be used, which provides the maximum field of view. For higher pressures (above about $970 \mathrm{kPa}$ ) a porthole window insert must be used, which has two smaller acrylic windows.

\section{Crossflow Instability and Transition on a Cone at Angle of Attack}

There are several instabilities that can cause a three-dimensional boundary layer to transition, including the centrifugal, streamwise, and crossflow instabilities. For an axisymmetric cone in hypersonic flow pitched at an angle of attack, the pressure is higher on the windward side than the leeward side, creating a circumferential pressure gradient. The pressure gradient causes the inviscid streamlines to be curved. A secondary flow (crossflow) perpendicular to the curved inviscid streamlines is created in the boundary layer because as you approach the wall, the pressure gradient remains constant while the streamwise velocity decreases. Since crossflow must vanish at the wall and the edge of the boundary layer, there is an inflection point in the crossflow velocity profile and therefore the crossflow is inviscidly unstable. ${ }^{6}$ The instability manifests as co-rotating vortices forming around the inflection point. The crossflow vortices can be either travelling or stationary with respect to the surface. It has been verified experimentally for low speeds that the stationary vortices tend to dominate in low-disturbance environments such as in flight or in low-noise tunnels, while the travelling vortices tend to dominate in high-disturbance environments such as conventional tunnels. ${ }^{7}$ It is not clear if these low speed results are also valid for high speed flows.

\section{A. Results}

Tests were performed in April 2012 to obtain global heat transfer from the temperature-sensitive paint on a $7^{\circ}$ half-angle cone at $6^{\circ}$ angle of attack. All experiments were performed in the BAM6QT under quiet flow. The model was equipped with six surface-mounted gauges along a single ray: 4 Schmidt-Boelter heat transfer gauges, and 2 PCB piezoelectric fast pressure transducers. The SB gauges were also equipped with two thermocouple readouts, at the base and surface of the sensor. Table A shows the position and the type of each sensor. The paint had an RMS roughness of $0.55 \mu \mathrm{m}$ and and the step at the leading edge of the paint had a slope of $10 \mu \mathrm{m} / \mathrm{mm}$. The method discussed in Reference 8 was used to feather the paint at the leading edge. Surface roughness measurements were made with a Mitutoyo SJ-301 surface roughness tester.

\begin{tabular}{ccccc}
\hline Position & $\begin{array}{c}\text { Distance from } \\
\text { Nosetip }\end{array}$ & $\begin{array}{c}\text { Gauge } \\
\text { Designation }\end{array}$ & $\begin{array}{c}\text { Heat Transfer } \\
\text { Calibration Range }\end{array}$ & $\begin{array}{c}\text { Gauge } \\
\text { Model }\end{array}$ \\
\hline \hline 1 & $0.149 \mathrm{~m}$ & $\mathrm{SB}-1$ & $0-22 \mathrm{~kW} / \mathrm{m}^{2}$ & $8-2-0.25-48-20835 \mathrm{TBS}$ \\
\hline 2 & $0.192 \mathrm{~m}$ & $\mathrm{SB}-2$ & $0-22 \mathrm{~kW} / \mathrm{m}^{2}$ & $8-2-0.25-48-20835 \mathrm{KBS}$ \\
\hline 3 & $0.235 \mathrm{~m}$ & $\mathrm{SB}-3$ & $0-11 \mathrm{~kW} / \mathrm{m}^{2}$ & $8-1-0.25-48-20835 \mathrm{TBS}$ \\
\hline 4 & $0.279 \mathrm{~m}$ & $\mathrm{PCB}-4$ & $\mathrm{~N} / \mathrm{A}$ & $132 \mathrm{~A} 31$ \\
\hline 5 & $0.321 \mathrm{~m}$ & $\mathrm{SB}-5$ & $0-11 \mathrm{~kW} / \mathrm{m}^{2}$ & $8-1-0.25-48-20835 \mathrm{TBS}$ \\
\hline 6 & $0.363 \mathrm{~m}$ & $\mathrm{PCB}-6$ & $\mathrm{~N} / \mathrm{A}$ & $132 \mathrm{~A} 31$ \\
\hline
\end{tabular}

Table 1. Sensor type and location.

The tests were conducted at a range of unit Reynolds numbers from $8.03 \times 10^{6}-12.0 \times 10^{6} / \mathrm{m}$. Measure- 
ments were made on the lee and yaw sides of the cone. The TSP was calibrated to heat transfer using the methods discussed in References 8 and 9. Good results were obtained using this calibration method with a cone in Mach-6 flow at $0^{\circ}$ angle of attack (the experimentally measured heat transfer was within $20 \%$ of the theoretical heat transfer.) The first or second Schmidt-Boelter gauge (SB-1 or SB-2) was used to calibrate the TSP. It proved to be easier to obtain a good fit between the calibrated TSP and the SB gauge if a stationary vortex was not present over the gauge, so the furthest upstream gauges were selected. Figure 2 shows four TSP images taken at increasing Reynolds numbers. At the lowest unit Reynolds number of $8.03 \times 10^{6} / \mathrm{m}(2(\mathrm{a}))$, the flow appears to be fully laminar. Crossflow vortices are faintly visible near the downstream end of the cone, but do not appear to be breaking down. Increasing the Reynolds number to $9.82 \times 10^{6} / \mathrm{m}(2(\mathrm{~b}))$, the crossflow vortices appear to grow in magnitude near the downstream end of the cone. It is possible that transition is occurring near the lee ray at this Reynolds number, but the TSP image is inconclusive.

Direct numerical simulations (DNS) have been performed at the University of Minnesota by Joel Gronvall for a $7^{\circ}$ cone at $6^{\circ}$ angle of attack. The simulations were performed at a Reynolds number of $9.5 \times 10^{6} / \mathrm{m}$ with no freestream disturbances. A randomly distributed roughness patch was placed near the nose along the windward ray. ${ }^{10}$ The DNS results in Figure $2(\mathrm{c})$ show that the stationary vortices appear to be breaking down to turbulence near the lee ray, qualitatively matching what is seen in the experiments. The DNS image in Figure 2(c) is at approximately the same conditions as the TSP image in Figure 2(b).

Returning to experimental results, increasing the Reynolds number to 10.6 and $12.0 \times 10^{6} / \mathrm{m}(3(\mathrm{a})$ and $3(\mathrm{~b})$ ) creates even larger stationary vortices. It also appears that transition is occurring near the lee ray. It is surprising that the stationary vortices amplify all the way to the lee side before breaking down to turbulence. $\mathrm{Li}$ et al. ${ }^{11}$ computed the maximum N-factors due to the stationary crossflow modes to be in excess of 20 at the downstream end of the cone at an azimuthal angle of approximately $130-140^{\circ}$ (where $0^{\circ}$ and $180^{\circ}$ correspond to the windward and leeward rays respectively). From the computations, it would be expected that the stationary vortices would break down closer to the yaw ray, but the experiments do not agree. The difference may be due to the non-linear breakdown necessary to cause transition.

On the lee side at these Reynolds numbers, the heat transfer $0.192 \mathrm{~m}$ downstream of the nosetip was between 0.54 and $0.66 \mathrm{~kW} / \mathrm{m}^{2}$. This is only $2.4-3.0 \%$ of the full scale of the SB gauges. Utilizing only a small percentage of the gauge's full scale may introduce substantial error in obtaining accurate heat transfer. Medtherm Corporation has cited two sigma uncertainty in the calibration of the SB gauge as $\pm 3 \%,{ }^{12}$ but the calibration is performed with a radiative heat source. The uncertainty of the gauge calibration under a convective heat flux condition (and a low level of convective heat flux) is not clear. Thus, the quantitative accuracy of the heat transfer values shown in Figures 2 and 3 is uncertain. Note that when the initial stagnation pressure is high enough, the smaller circular porthole windows must be used, reducing the field of view.

Figure 4 shows the heat transfer from SB-2 during the same run as the TSP image in Figure 2(a), over the course of a run, along with the calibrated TSP at a comparison patch (this comparison patch should see nominally the same heat transfer as the SB gauge). The coefficient of determination between the two data sets is 0.951 . The curve fit gives a decent calibration at these low heat transfer on the lee side, but better calibrations are typically obtained when the heat transfer is higher. ${ }^{9}$

Figure 5 shows surface-pressure power spectra for the four Reynolds numbers, taken with the two PCB gauges installed flush with the model. The gauges were installed along the lee ray of the cone. At the lowest Reynolds number (blue curves), there appears to be an instability at approximately $62 \mathrm{kHz}$ that begins growing near PCB-6. Increasing the Reynolds number to $9.82 \times 10^{6} / \mathrm{m}$ (green curves), the instability appears further upstream, shown by the peak in the spectra at $82 \mathrm{kHz}$ at PCB-4. The peak appears to be broadening, suggesting that transitional flow may be occurring near PCB-4. PCB-6 yields data indicative of transitional flow, as the spectra show a broadband increase in power, and the peak at $82 \mathrm{kHz}$ is no longer present. Once again increasing the Reynolds number (red curves), the peak in the spectra at PCB-4 has moved to $94 \mathrm{kHz}$, and the peak appears to have broadened with the increase in Reynolds number. The increase of the peak frequency with Reynolds number seems to suggest that the instability may be a second-mode wave, as the frequency of these waves are inversely proportional to boundary layer thickness. Moving to the highest Reynolds number (black curves), the spectra at PCB-4 shows a very broad peak near $126 \mathrm{kHz}$, suggesting that transition may be occurring closer to PCB-4. The TSP image (3(b)) also shows what appears to be transitional flow at the axial location of PCB-4.

Tests were also performed with the yaw side of the cone in view, along with the sensors installed along 


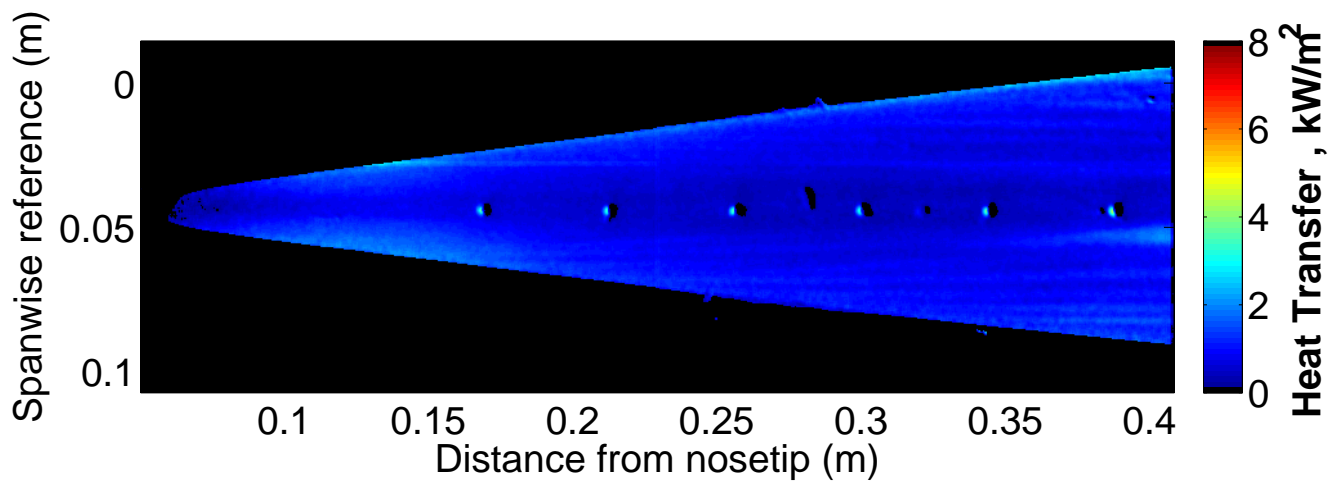

(a) $p_{0}=105.8$ psia, $\operatorname{Re}=8.03 \times 10^{6} / \mathrm{m}, T_{0}=425.2 \mathrm{~K}, T_{w}=296.7 \mathrm{~K}$

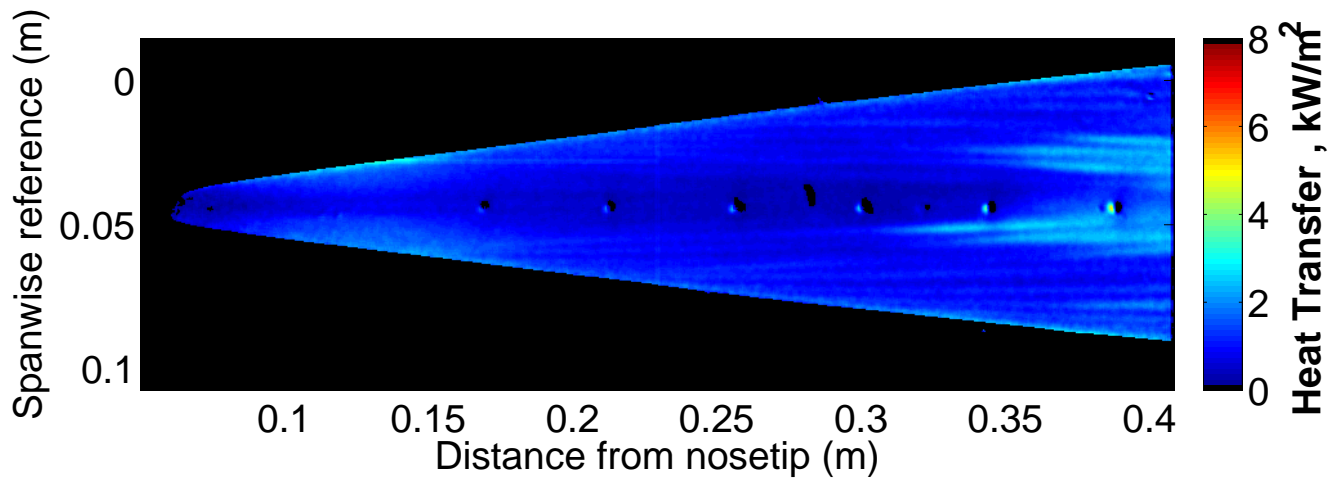

(b) $p_{0}=129.5 \mathrm{psia}, \operatorname{Re}=9.82 \times 10^{6} / \mathrm{m}, T_{0}=425.2 \mathrm{~K}, T_{w}=301.2 \mathrm{~K}$

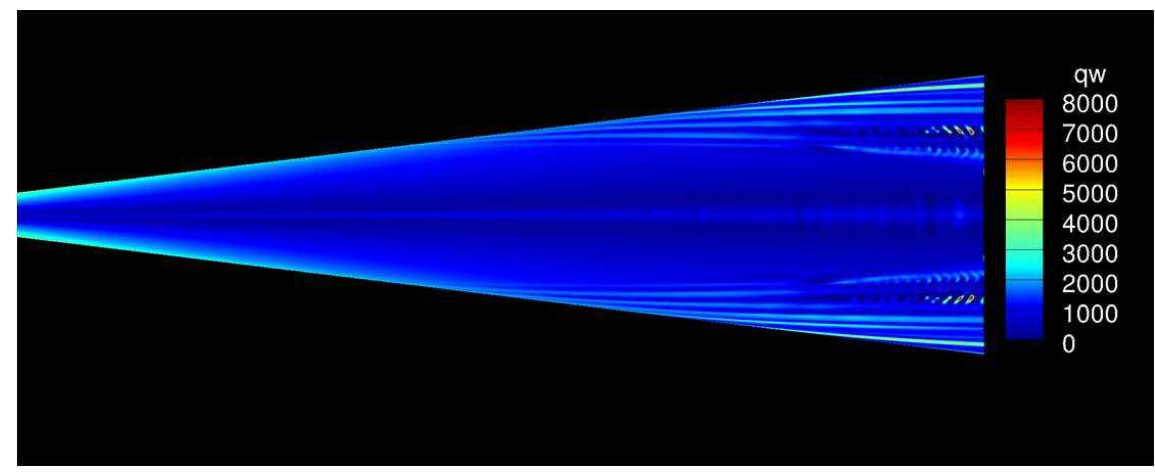

(c) DNS image from Reference 10. $p_{0}=134.4 \mathrm{psia}, \operatorname{Re}=9.5 \times 10^{6} / \mathrm{m}, T_{0}=433 \mathrm{~K}$, $T_{w}=300 \mathrm{~K}$

Figure 2. TSP and DNS images of the $7^{\circ}$ half-angle cone at $6^{\circ}$ angle of attack at lower Reynolds numbers. Lee side of the cone. Quiet flow. 


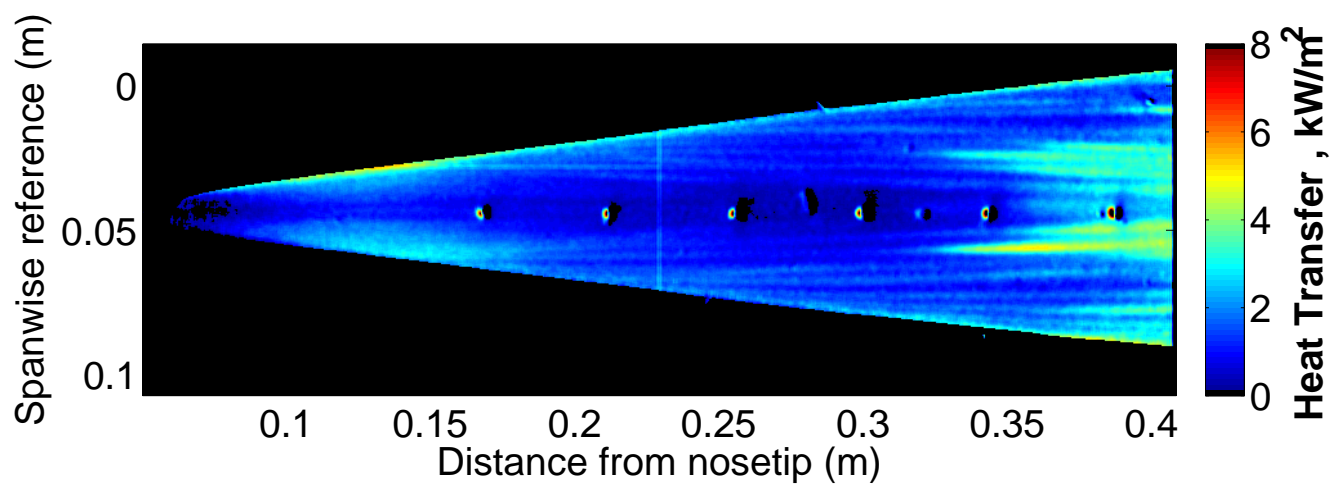

(a) $p_{0}=139.5 \mathrm{psia}, \mathrm{Re}=10.6 \times 10^{6} / \mathrm{m}, T_{0}=424.5 \mathrm{~K}, T_{w}=296.7 \mathrm{~K}$

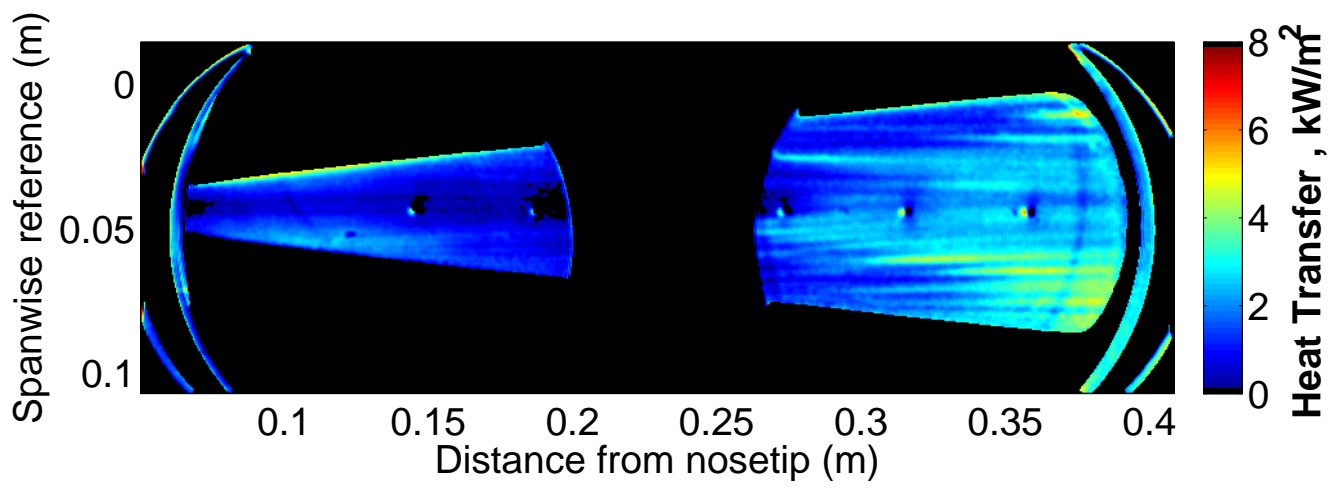

(b) $p_{0}=158.0 \mathrm{psia}, \operatorname{Re}=12.0 \times 10^{6} / \mathrm{m}, T_{0}=424.4 \mathrm{~K}, T_{w}=299.9 \mathrm{~K}$

Figure 3. TSP and DNS images of the $7^{\circ}$ half-angle cone at $6^{\circ}$ angle of attack at higher Reynolds numbers. Lee side of the cone. Quiet flow. 


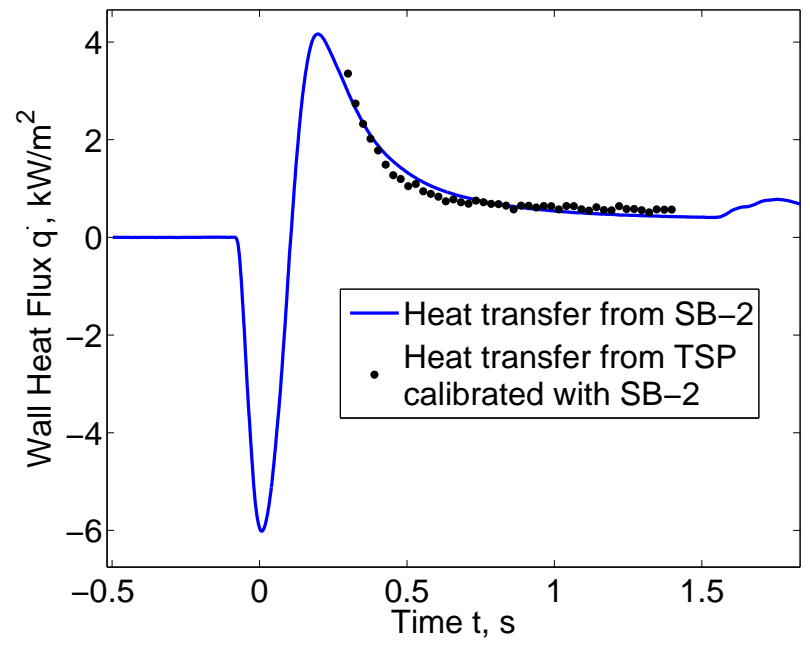

Figure 4. Plot of heat transfer from SB-2 along with heat transfer calculated from a comparison patch of TSP near the sensor. Same run as the TSP image in Figure $2(a)$. Re $=8.03 \times 10^{6} / \mathrm{m}$, lee side of cone.

the yaw ray. The TSP images calibrated to heat transfer are shown in Figure 6. At the lowest Reynolds number, the crossflow vortices are only faintly visible. Increasing the Reynolds number creates slightly higher-amplitude stationary vortices, but they do not appear to be breaking down to turbulence on the yaw side of the cone. In the DNS image (6(e)), the crossflow vortices are visible but do not begin to break down to turbulence, once again qualitatively agreeing with the experimental results. The DNS image in Figure 6(e) is at approximately the same conditions as the TSP image in Figure 6(b). At the highest experimental unit Reynolds number (6(d)), once again the stationary vortices are stronger, but transition still does not appear to be occurring on the yaw side. On the yaw side at these unit Reynolds numbers, the heat transfer at $0.192 \mathrm{~m}$ was between 1.70 and $2.14 \mathrm{~kW} / \mathrm{m}^{2}$. This is $7.7-9.7 \%$ of the full scale of the SB gauges, which is still a small percentage but may allow for more accurate heat transfer measurements on the yaw side as compared to the lee side.

Surface-pressure power spectra for the yaw-side data at the four Reynolds numbers are shown in Figure 7. The PCB gauges were installed along the yaw ray of the cone. There are no clear peaks in the spectra. Perhaps the PCB gauges were not able to measure the travelling crossflow waves. 


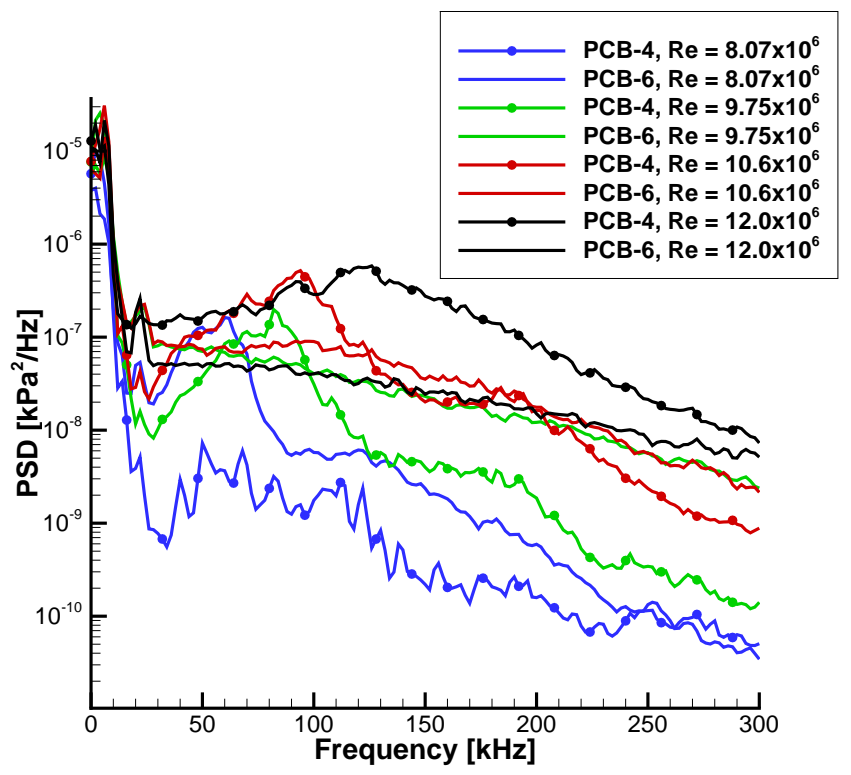

Figure 5. Power spectra of surface pressure at $0.279 \mathrm{~m}(\mathrm{PCB}-4)$ and $0.363 \mathrm{~m}(\mathrm{PCB}-6)$ from the nosetip, at four different Reynolds numbers. Sensors along the lee ray. Quiet flow.
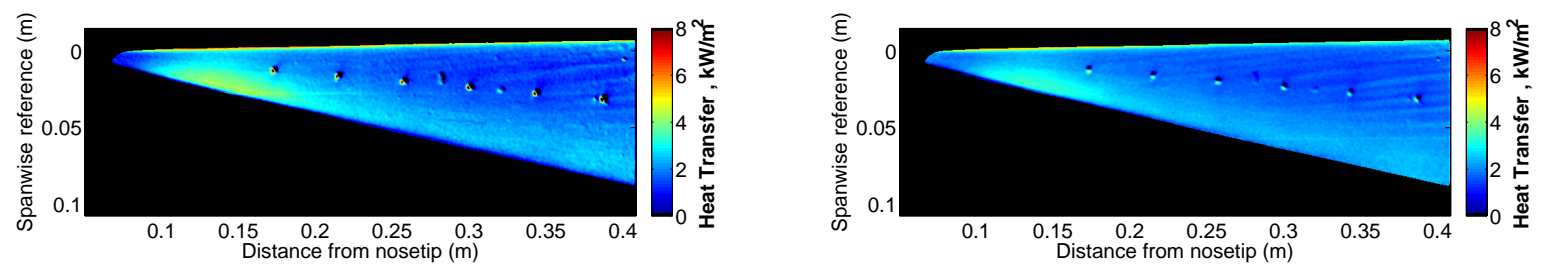

(a) $p_{0}=106.5$ psia, $\operatorname{Re}=8.07 \times 10^{6} / \mathrm{m}, T_{0}=425.3 \mathrm{~K},(\mathrm{~b}) p_{0}=128.5 \mathrm{psia}, \operatorname{Re}=9.75 \times 10^{6} / \mathrm{m}, T_{0}=425.0 \mathrm{~K}$, $T_{w}=296.4 \mathrm{~K}$ $T_{w}=303.1 \mathrm{~K}$
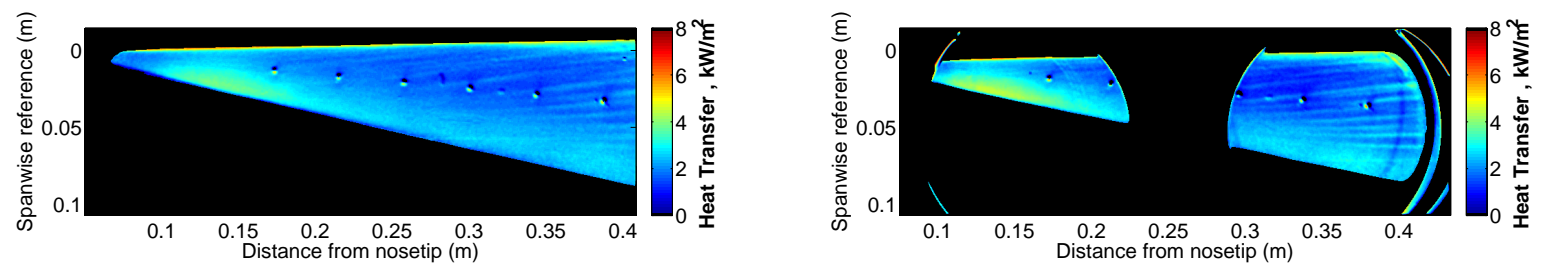

(c) $p_{0}=139.5$ psia, $\operatorname{Re}=10.6 \times 10^{6} / \mathrm{m}, T_{0}=424.6 \mathrm{~K}$, (d) $p_{0}=158.1$ psia, $\operatorname{Re}=12.0 \times 10^{6} / \mathrm{m}, T_{0}=424.5 \mathrm{~K}$, $T_{w}=302.1 \mathrm{~K}$ $T_{w}=297.5 \mathrm{~K}$

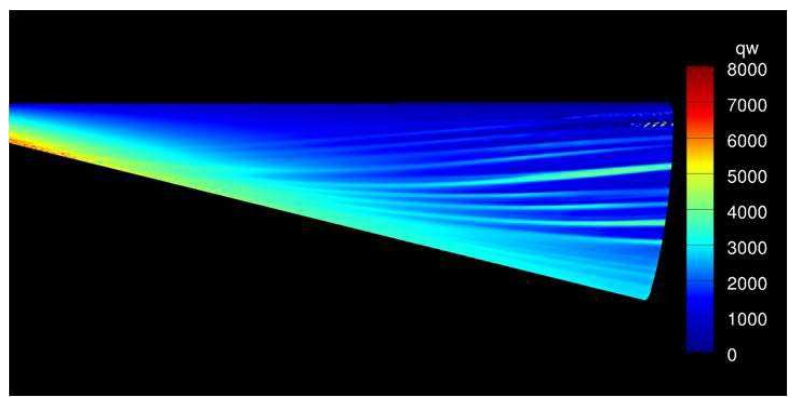

(e) DNS image from Reference 10. $p_{0}=134.4$ psia, $\operatorname{Re}=9.5 \times 10^{6} / \mathrm{m}, T_{0}=433 \mathrm{~K}, T_{w}=300 \mathrm{~K}$

Figure 6. TSP images of the $7^{\circ}$ half-angle cone at $6^{\circ}$ angle of attack. Yaw side of the cone. Quiet flow. 


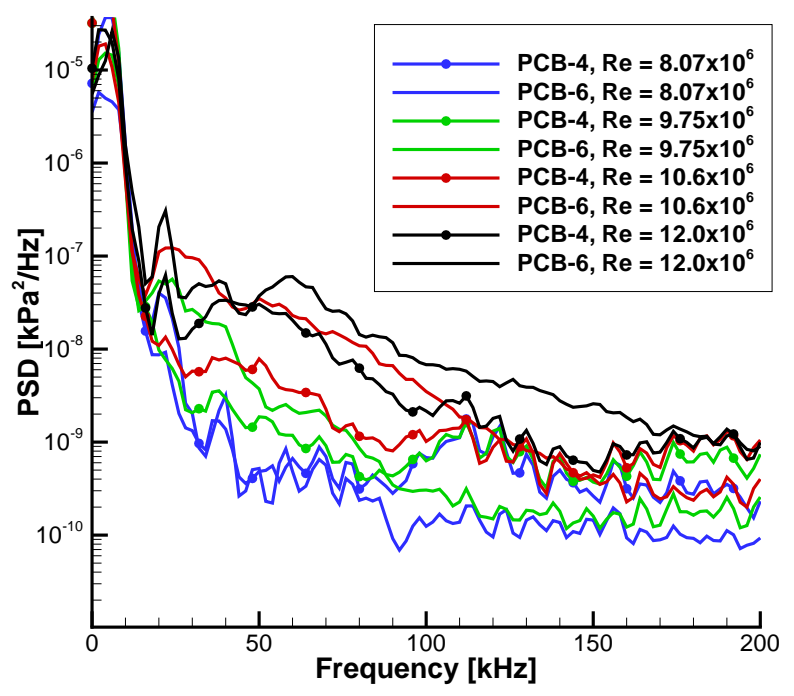

Figure 7. Power spectra of surface pressure at $0.279 \mathrm{~m}(\mathrm{PCB}-4)$ and $0.363 \mathrm{~m}(\mathrm{PCB}-6)$ from the nosetip, at four different Reynolds numbers. Sensors along the yaw ray. Quiet flow. 


\section{Interruption of Streak Heating on a Flared Cone Via Controlled Spanwise-Periodic Roughness Elements}

Select results are presented from the ongoing effort ${ }^{8}$ to manipulate the streamwise streaks characteristic of the flared cone transition process under quiet flow using discrete roughness element arrays. Part of the difficulty lies in creating roughness elements (dots) whose size and position on the cone can be readily controlled in a cost- and time-effective manner.

Dots made from single-component epoxy are one plausible option. The epoxy is heat-cured in as little as 5 minutes and has unlimited working life, which allows for ample time to apply dots. The epoxy is single component, which ensures that all dots will cure, regardless of size. Once cured, the epoxy can withstand temperatures up to $475 \mathrm{~K}$, making it suitable for use in the BAM6QT.

The dots are applied using an Electronic Fluid Dispenser (EFD). Epoxy is transferred to a syringe which connects to the EFD via an adapter cap and tube. The EFD regulates high pressure air into the syringe, which can be equipped with different types and sizes of dispense tips. The EFD also controls the time over which an air pulse is delivered, triggered by the user. The size of a dot can be controlled by varying output pressure, dispense time, dispense tip gauge, and epoxy viscosity.

The dots used in these experiments had an average diameter of $0.050 \mathrm{in}$. and an average height of 0.020 inches. The standard deviation was within $0.001 \mathrm{in}$. for both diameter and height, based on a sample size of 7 dots. The dots were placed at an axial location of roughly 10.04 in., on the cone's roughness insert. According to STABL mean flow computations at 140 psia, the boundary layer thickness at this location is approximately 0.035 inches.

The dots were applied by mounting the roughness insert to a rotary stage, which allows for angular accuracy better than 1/10th of a degree. The epoxy syringe was mounted in a stand, maintaining position relative to the insert. This allows a dot to be precisely placed at any angle relative to an arbitrary baseline.

First, 30 dots were placed around the circumference of the insert. To test a wider spacing, every other dot was scraped off, leaving 15 dots around the insert. The spaces between the remaining dots were cleaned with acetone to eliminate any roughness from epoxy residue. Figure 8(a) shows the heat transfer for the 30-dot case. All data are for quiet flow. There is a noticeable lack of streak heating; instead, vortex pairs can be seen downstream of each dot. Analysis of the spanwise heat transfer profile shows a regular spacing of $12^{\circ}$ between each vortex pair, which corresponds to an azimuthal wavenumber of 30 .

Figure 8(b) shows an average of 6 axial heat transfer profiles taken along random rays. There is a constant increase in heat transfer up to approximately $17.5 \mathrm{in}$., after which the heat transfer rate plateaus, indicating the end of transition. The SB gauge at 20.4 in. measured heat transfer rates comparable to those measured under typical conditions with no added roughness. The end of transition is confirmed in the power spectra shown in Figure 8(c), which shows broadband pressure fluctuations starting at 17.5 inches. The spectra also show a peak near $285 \mathrm{kHz}$, which is at a lower frequency than the $300 \mathrm{kHz}$ typical for this pressure. More importantly, the peak amplitude is roughly 100 times smaller than what is typical, and there is no harmonic. This indicates that the dots are damping the second-mode waves.

Figure 9 shows heat transfer and spectra data from the 15 dot case. Evidently the 15 dot spacing is wide enough to allow streak heating between the vortex pairs. The wedge angle of these trailing vortex pairs was measured in TSP images to be approximately $13^{\circ}$. The pressure sensors were positioned between dots, and confirm the non-linear growth of second-mode waves in the power spectra. The fundamental peak in the case with dots is shifted down by about $10 \mathrm{kHz}$ to $290 \mathrm{kHz}$, but the amplitude is roughly equal to the typical case. This implies that when the dots are spaced far enough apart, they have an effect on the most amplified frequency, but not necessarily its amplitude.

These cases show that these particular dots do not accomplish the intended goal, which is to manipulate streak heating. The dots are large enough that they override the natural second-mode wave induced transition process, causing instead what appears to be roughness-induced transition due to vortex formation. This method will have to be improved before it is possible to manipulate streak spacing and other properties without bypassing second-mode wave induced transition. The first step would be to try to make smaller dots with the available epoxy. It was possible to make dots approximately $15 \%$ smaller than the ones used in these cases, but they were not as uniform. Another option would be to obtain a lower viscosity epoxy. This should allow small dots to be made without a loss in uniformity. These results and related work will appear in an upcoming Master's thesis. ${ }^{13}$ 


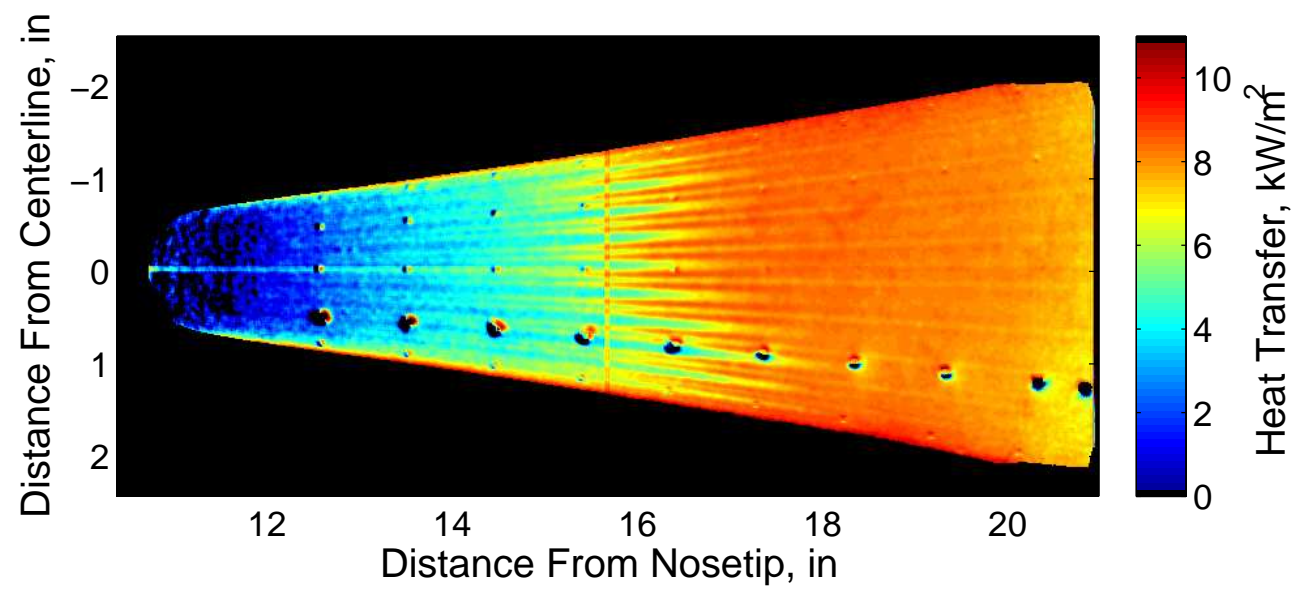

(a) Global heat transfer

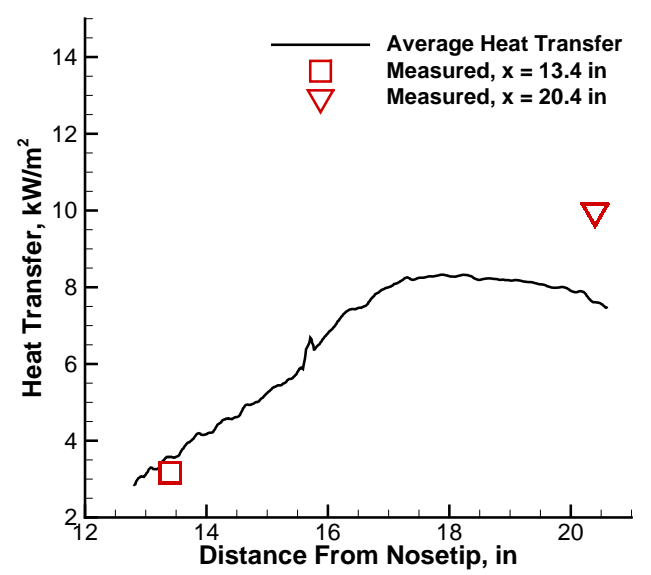

(b) Axial heat transfer profile

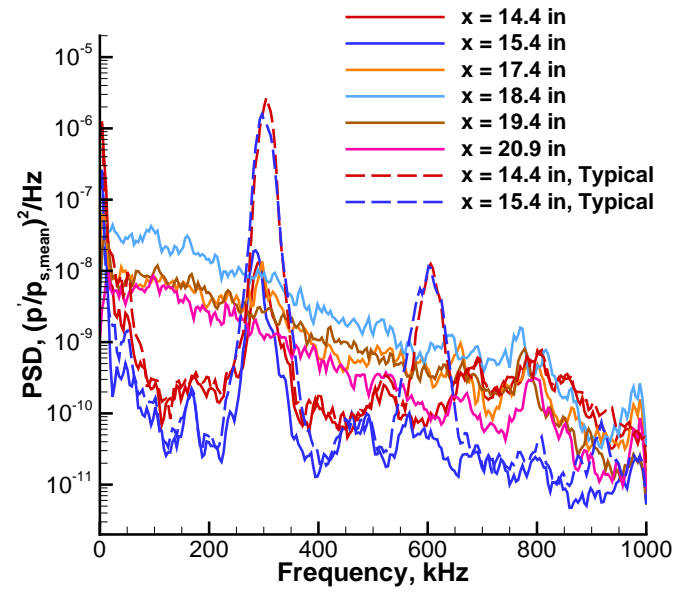

(c) Power spectra

Figure 8. Heat transfer and power spectra with 30 epoxy dots, quiet flow, $p_{0} \approx 140$ psia 


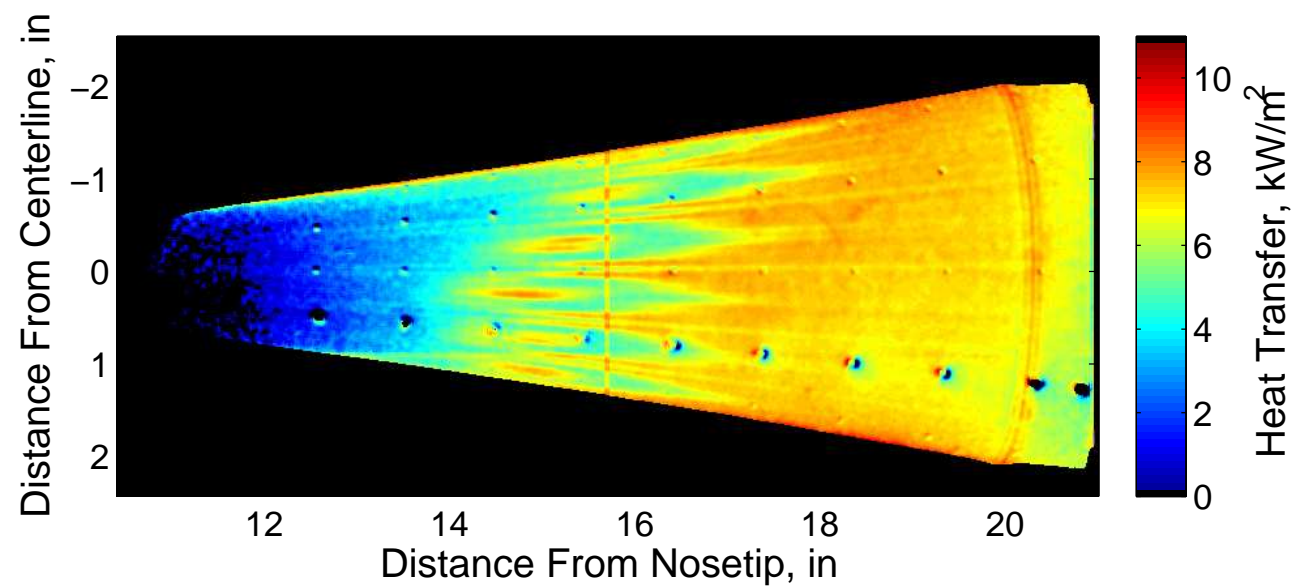

(a) Heat transfer

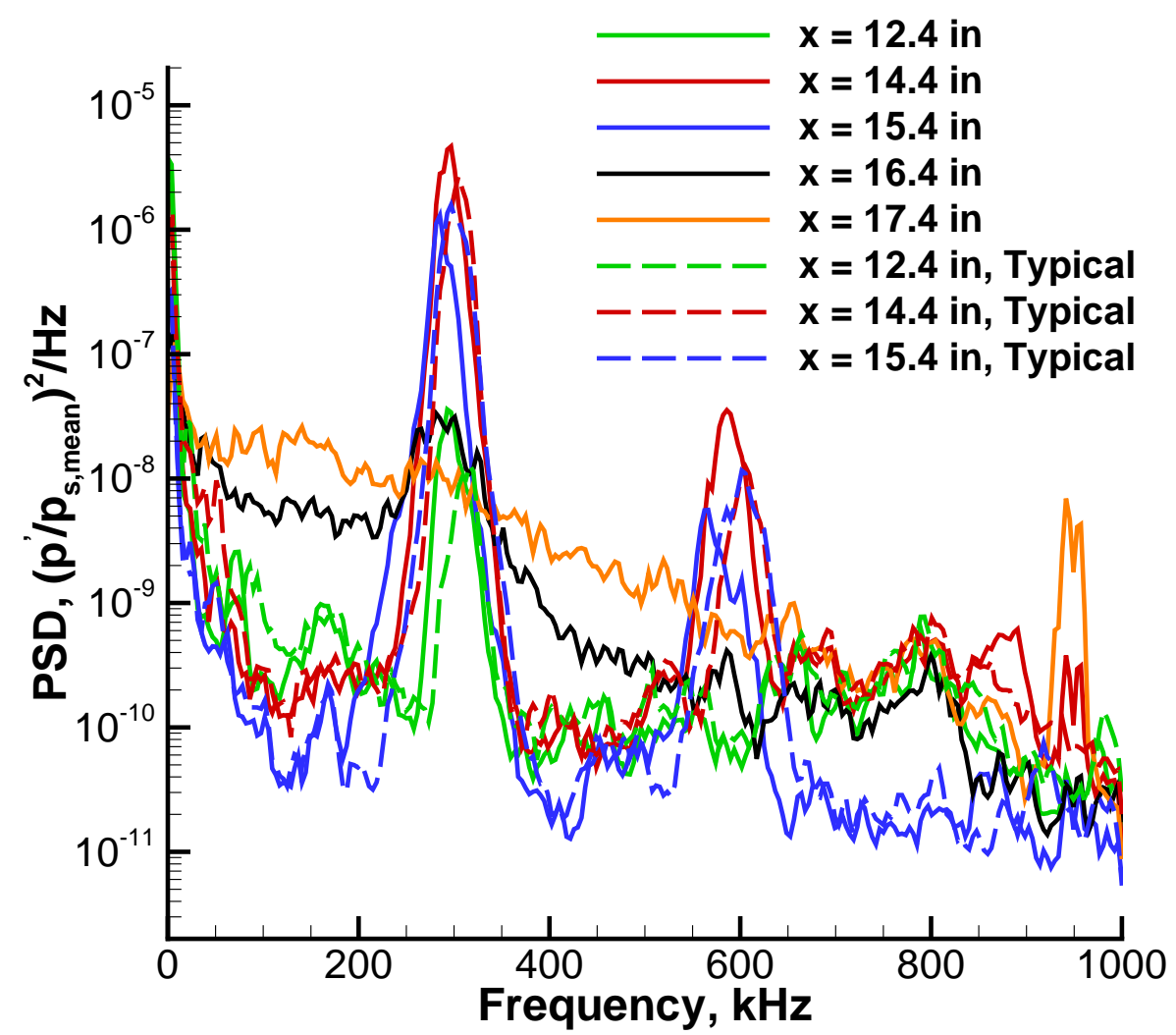

(b) Power spectra

Figure 9. Heat transfer and power spectra with 15 epoxy dots, quiet flow, $p_{0} \approx 140$ psia 


\section{Forward-Facing Cavity}

A forward-facing cavity (Figure 10) is used as a means of showing the laser perturber apparatus works in the BAM6QT. The physical response of the model is fairly well-known from previous experiments in the Mach-4 Purdue Quiet Flow Ludwieg Tube (PQFLT). ${ }^{14,15}$ Furthermore, the large-diameter nose facilitates the alignment of this model with the freestream laser perturbation. Computations have also been done on similar geometries by Engblom. ${ }^{16}$

\section{A. Theory}

The forward-facing cavity acts as a resonance tube, where the pressure fluctuations within the cavity have a fundamental resonant frequency of

$$
\omega_{1 n}=2 \pi f_{1 n}=\frac{\pi a_{0}}{2 L^{*}}
$$

where $a_{0}$ is the speed of sound based on stagnation temperature in the cavity and $L^{*}=L+\delta$ is the axial distance between the cavity base and mean shock location. The mean shock standoff distance $\delta$ can be calculated numerically, measured experimentally, or estimated using existing correlations. However, no correlation currently exists for a forward-facing cavity. The shock standoff distance was calculated by averaging the correlation for that of a flat-nosed cylinder and that of a sphere. At $M=6, \delta=0.54 D$ for a flat-nosed cylinder and $\delta=0.14 D$ for a sphere. The average of these two expressions gives $\delta=0.34 D$ for the forward-facing cavity. The constants $D_{\text {nose }}$ and $D$ are as noted in Figure 10.

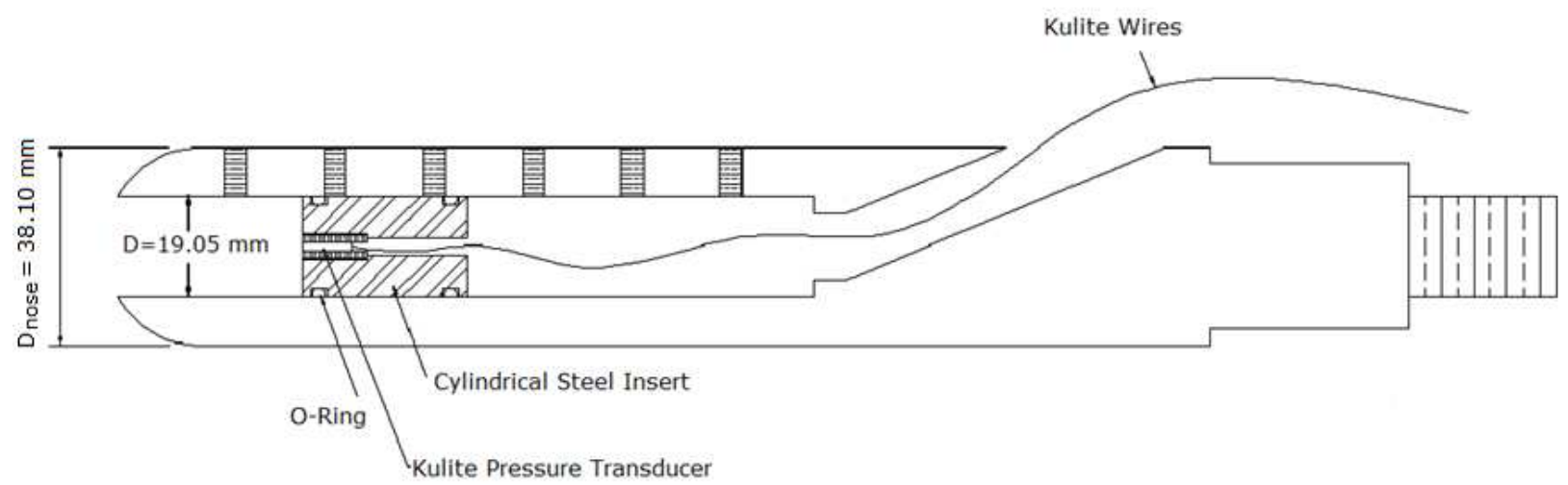

Figure 10. Schematic of forward-facing cavity model.

At some critical depth, a forward-facing cavity will create self-sustained resonance, where a deep cavity resonates strongly, regardless of the amplitude of freestream noise. Previous experiments showed the pressure fluctuations within the cavity are three orders of magnitude larger than when the cavity depth is shallow. ${ }^{8}$ In 2008, Segura found that the critical cavity depth was about $L / D=1.2$ in the PQFLT. ${ }^{15}$ The critical depth in the BAM6QT was found to be the same $(L / D=1.2)$ in $2011 .^{8}$

\section{B. Model and Instrumentation}

The model used for these experiments (Figure 10) was one designed by Segura in 2008. ${ }^{17}$ The maximum depth possible for this model is $L / D=5.00$. The depth was varied by sliding the cylindrical steel insert back and forth, and held constant by tightening set screws on the side of the model. The cavity is sealed at the base using two o-rings on the cylindrical steel insert.

Pressure fluctuations in the cavity were measured with a B-screen Kulite XCQ-062-15A pressure transducer mounted in the base of the cavity, within the cylindrical steel insert. These sensors are mechanically stopped at pressures above 15 psia to prevent damage to the transducer. The particular sensor used in this experiment has a resonant frequency of about $270 \mathrm{kHz}$. The data were sampled at $1 \mathrm{MHz}$ for 5 seconds. However, the Kulite transducers are reported to have flat frequency response only up to about one-fifth of 
the resonant frequency. This limitation is not a problem for these experiments because the cavity resonant frequencies are less than $30 \mathrm{kHz}$.

The optical system used consists of three lenses and a 1.5-inch-diameter window for optical access into the BAM6QT. For these preliminary tests, a flat window was used. Since this window does not share the same contour as the axisymmetric BAM6QT test section, some disturbances may be introduced in the tunnel. Work to characterize these disturbances is ongoing. The lenses used are air-spaced YAG triplets manufactured by CVI Melles-Griot. In order from laser head to BAM6QT, these lenses are:

- a YAN-50-10 $\left(f_{\text {eff }}=-50 \mathrm{~mm}\right)$ to expand the laser beam

- a YAP-200-40 $\left(f_{\text {eff }}=+200 \mathrm{~mm}\right)$ to collimate the laser beam

- a YAP-200-40 $\left(f_{\text {eff }}=+200 \mathrm{~mm}\right)$ to focus the laser beam

where $f_{\text {eff }}$ denotes the effective focal length of each triplet. The thickness of the BAM6QT window is nominally $46.8 \mathrm{~mm}$.

The laser used with this optical system is a Spectra-Physics Quanta-Ray GCR-190-10. It is a seeded, frequency-doubled Nd:YAG laser that operates at $10 \mathrm{~Hz}$. Each pulse lasts about $7 \mathrm{~ns}$ and is capable of having a maximum energy of $300 \mathrm{~mJ}$. The spatial profile of the beam has a 90\% Gaussian fit.

As in Ladoon's experiments, ${ }^{14}$ a laser-generated freestream perturbation was created upstream of the forward-facing cavity model. In the BAM6QT, the onset of uniform flow occurs at $z=1.914 \mathrm{~m}$, where $z=0$ corresponds to the axial location of the throat. The laser perturbation is created on the centerline of the tunnel at $z=1.925 \mathrm{~m}$. This position is limited by the optical design of the perturber as well as the optical access into the tunnel. Only one set of windows of acceptable optical quality exists, and this set of windows is centered at $z=1.924 \mathrm{~m}$. The perturber optics were aligned to create a slight angle between the window and the perturber optics, to reduce the risk of damage from back reflections.

\section{Effect of Distance on Laser Perturbation Detection}

It is critical to position the model such that the laser perturbation is measurable at the model location. If the model is too far away from the position at which the perturbation is created, the perturbation may not be detected. Furthermore, if the perturbation is created off-axis of the model, the perturbation may not be detected. Initially, the forward-facing cavity was positioned so that $z_{\text {nose }}=2.374 \mathrm{~m}$. This was to correspond to many pitot-probe measurements that had been made at the same location. However, this location proved to be too far from the perturbation, as seen in Figure 11(a), which shows the cavity base pressure as measured by a Kulite in the top plot, in blue. The lower plot shows the Q-switch sync signal output by the laser when a high-powered pulse is fired from the laser head. When a high-powered pulse is fired, the Q-switch sync signal outputs $2.0 \mathrm{~V}$. A perturbation is created almost immediately following (about $1 \mathrm{~ns}$ after) the high-powered pulse. Two turbulent spots pass along the nozzle wall at about $t=1.30$ and $1.35 \mathrm{~s}$. As shown, no perturbations are visible in the cavity base pressure, corresponding to the laser pulses.

However, when the model is moved closer to the perturbation by an arbitrary amount (to $z=1.979 \mathrm{~m}$ ), the perturbation becomes detectable. In Figure 11(b), a spike in the measured cavity base pressure occurs each time the Q-switch sync signal reaches $2.0 \mathrm{~V}$. This indicates that every time a high-powered pulse is fired, a perturbation is created, and this perturbation is detected by the Kulite mounted in the base of the forward-facing cavity.

Testing is currently being done to find the maximum distance at which the model could be placed from the perturbation while still being able to detect the perturbation. It is possible that the perturbation is not created on-axis with the forward-facing cavity model and therefore does not convect in a straight line toward the model. Further investigation of this phenomenon must be conducted.

\section{Minimum Freestream Density with Detectable Laser Perturbations}

The arbitrary location of $z_{\text {nose }}=1.979 \mathrm{~m}$ was used to detect the lowest density at which a laser perturbation could be created. This is not ideal, as part of the model is in non-uniform flow. However, for the purposes of determining that the laser perturber system worked, the location suffices. Several runs at different initial stagnation pressures were made. For initial stagnation pressures less than $480 \mathrm{kPa}$, a 12-inch ball valve was used to start the tunnel. This method of operating the BAM6QT is explained in Reference 18. All other runs used the double-burst-diaphragm system that is typically used to start the tunnel. 

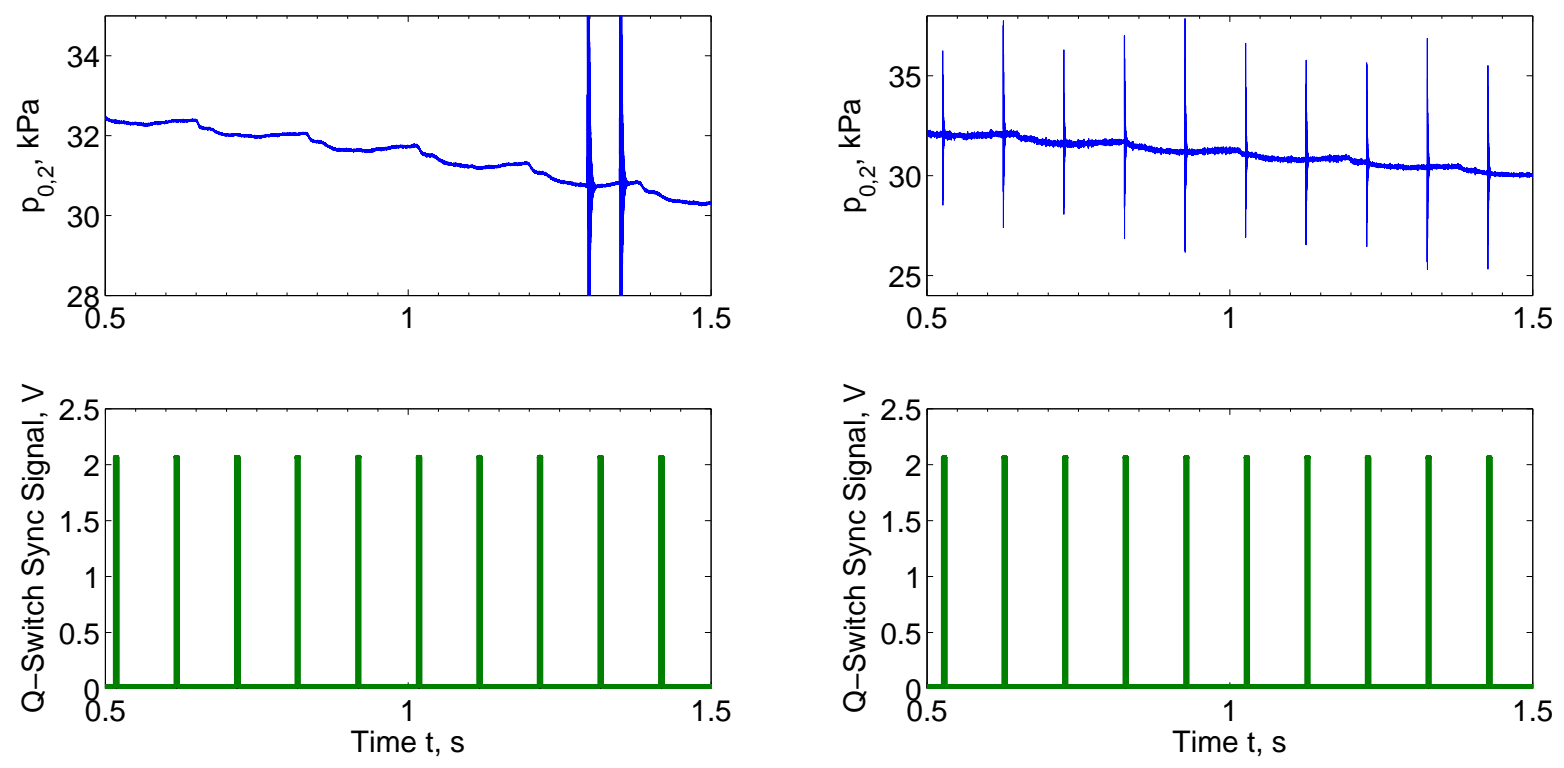

(a) $z_{\text {nose }}=2.374 \mathrm{~m} . L / D=1.00$, quiet flow, $p_{0, i}=1129 \mathrm{kPa}$, $T_{0, i}=161.2^{\circ} \mathrm{C}$. No laser perturbations.

(b) $z_{\text {nose }}=1.979 \mathrm{~m} . L / D=1.00$, quiet flow, $p_{0, i}=1130 \mathrm{kPa}$, $T_{0, i}=162.3^{\circ} \mathrm{C}$. Laser perturbations present.

Figure 11. Detection of perturbations are dependent on axial location of model.

Two of the runs are shown in Figure 12. Note that the vertical axes for Figure 12(a) are an order of magnitude larger than for Figure 12(b). Figure 12(a) shows the typical response seen in higher density flow. When the density is reduced (Figure 12(b)), the initial amplitude is barely larger than the natural cavity resonance. Without the high-frequency noise associated with Kulite sensor resonance, it is difficult to distinguish the response as a response to the laser perturbation. In fact, this initial amplitude is so small that it is possible that the high-frequency Kulite response may be due only to electronic noise from the laser output.

To determine if the Kulite response was due to the laser perturbation interacting with the model bow shock, the case with the lowest freestream density (Figure 12(b)) was compared to an electronic noise trace (Figure 12(c)). The electronic noise trace (Figure 12(c)) was taken when there was no flow in the tunnel at an ambient pressure of $p=1.10 \mathrm{kPa}$. The laser power supply was turned on and the flash lamps were allowed to simmer. (No laser light escaped the laser head, but the flash lamps were on.) A comparison of the lowest-density trace to the electronic noise trace shows that the Kulite response is more likely due to some flow phenomenon.

The presence of natural cavity resonance can also make it difficult to tell whether a small laser perturbation has interacted with the cavity. At densities less than $0.011 \mathrm{~kg} / \mathrm{m}^{3}$, perturbations could not be distinguished from the natural cavity resonance. Thus, the minimum freestream density at which laser perturbations can still be detected in a forward facing cavity is $\rho_{\infty}=1.07 \times 10^{-2} \mathrm{~kg} / \mathrm{m}^{3}$.

At a laser pulse energy of only $180 \mathrm{~mJ} /$ pulse, the minimum density measured is much lower than previously measured in a test cell. ${ }^{19,20}$ Figure 13 shows that the threshold minimum density at which perturbations were detected in the test cell for both sets of perturber optics is very similar. The PQFLT optics system tested by Schmisseur in 1994 is given by red squares. Data for the BAM6QT optics system is given by blue triangles. However, the minimum density at which a perturbation was detected in the BAM6QT (green star in Figure 13) is about $60 \%$ of the threshold minimum density in the test cell for the given laser energy. The reason for this is not yet known. 


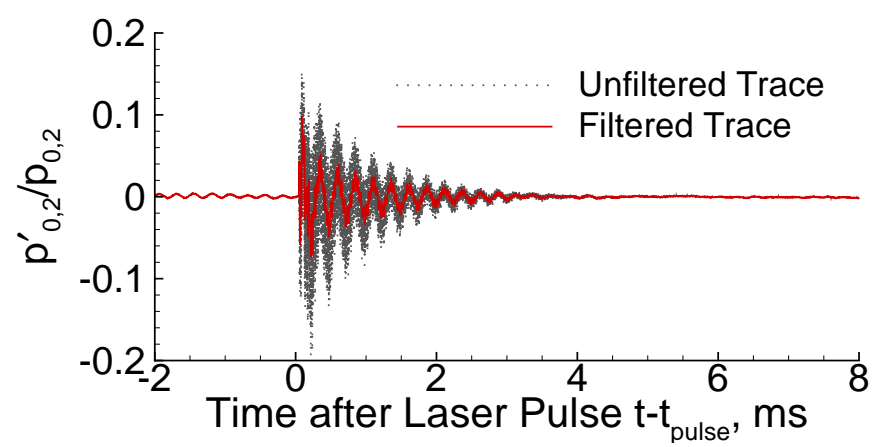

(a) $R e_{\infty} / \mathrm{m}=11.17 \times 10^{6} / \mathrm{m} . p_{0, i}=1130 \mathrm{kPa} . \rho_{\infty}=0.0428 \mathrm{~kg} / \mathrm{m}^{3}$.

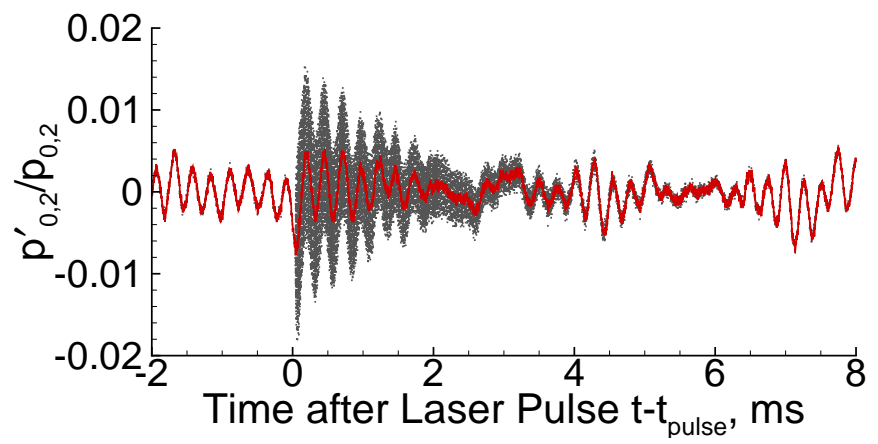

(b) $R e_{\infty} / \mathrm{m}=2.84 \times 10^{6} / \mathrm{m} . \quad p_{0, i}=289.6 \mathrm{kPa} . \quad \rho_{\infty}=1.07 \times$ $10^{-2} \mathrm{~kg} / \mathrm{m}^{3}$. Lowest density at which perturbation is detected.

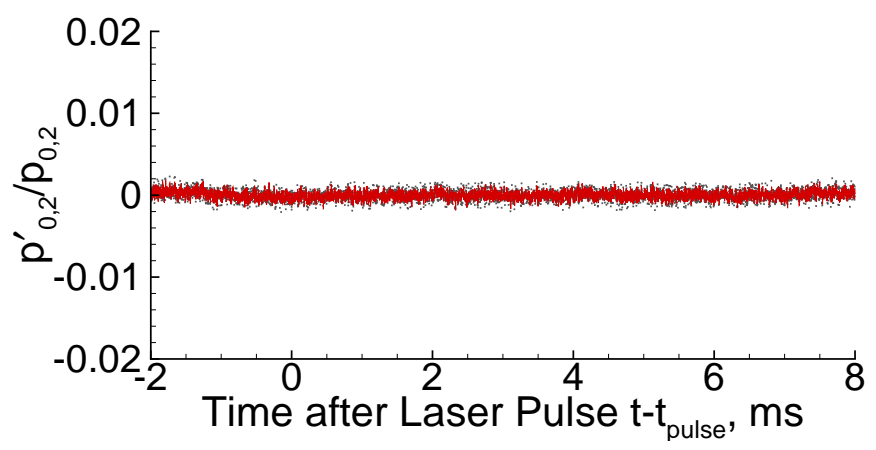

(c) Electronic noise trace. No flow. $\rho=0.0134 \mathrm{~kg} / \mathrm{m}^{3}$.

Figure 12. A comparison of base cavity pressure at different freestream densities. $L / D=1.00 . \quad E_{\text {pulse }}=$ $180 \mathrm{~mJ} /$ pulse. Quiet flow.

\section{Future Work}

Additional tests will be performed with this model. First, the maximum distance possible between the laser perturbation and the model must be determined. This will help in the positioning of other future models for receptivity studies. Second, the damping characteristics of the interaction between the laser perturbation and the forward-facing cavity must be investigated at a location where the entire model is in uniform flow. Damping characteristics at the location of $z_{\text {nose }}=1.979 \mathrm{~m}$ are available but occur at a location where the model is not in uniform flow. This lack of uniform flow could have corrupted some of the characteristics and needs to be further investigated. Computations for this model are also desired, for comparison to the experiment. After these studies are completed, the laser perturber will be tested with a flared cone for receptivity studies. 


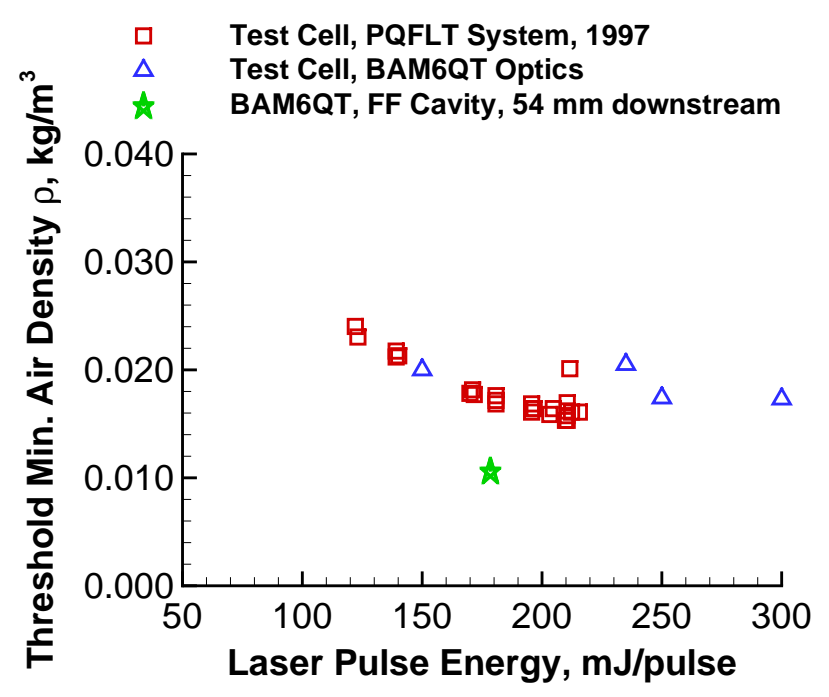

Figure 13. Comparison of lowest density at which laser perturbation is seen in BAM6QT and in test cell.

\section{Investigation of Hypersonic Corner Flow Transition on a $7^{\circ}$ Half Angle Straight Cone}

Supersonic corner flow has been studied since the 1960's. Stainback found that the flow field was characterized by a pair of vortices running along each side of the corner. ${ }^{21,22}$ These vortices were accompanied by a cooler region in the proximity of the corner. Korkegi found that a strong enough shock could form a secondary vortex within the flowfield. ${ }^{23}$ Transition of these flows has not been investigated at hypersonic Mach numbers.

Hypersonic corner flow transition is an important parameter in the design of modern two dimensional scramjets, such as the $\mathrm{X}-51 .{ }^{24}$ The $\mathrm{X}-51$ was designed with the assumption that the boundary-layer entering the scramjet would be turbulent. A turbulent boundary-layer increases mixing within the combustor and prevents engine unstart from shock/boundary-layer interactions within the isolator.

An existing $7^{\circ}$ half-angle straight cone was modified to add a perpendicular fin to the cone frustum. This modification allowed for a preliminary studies of corner flow transition in the hypersonic regime. Fig 14 illustrates the modifications made to the cone. The leading edge of the fin was swept by $10^{\circ}$ to prevent a detached shock from forming, and the entire fin was kept within the bow shock from the nose tip of the cone. The junction between the fin and the frustum forms an interior angle of $37^{\circ}$ to better represent the inlet cowling of the X-51. TSP measurements were made to investigate the flow between $\operatorname{Re}=7.70-11.83 \times 10^{6} / \mathrm{m}$ at a $\mathrm{T}_{o}=160^{\circ} \mathrm{C}$.

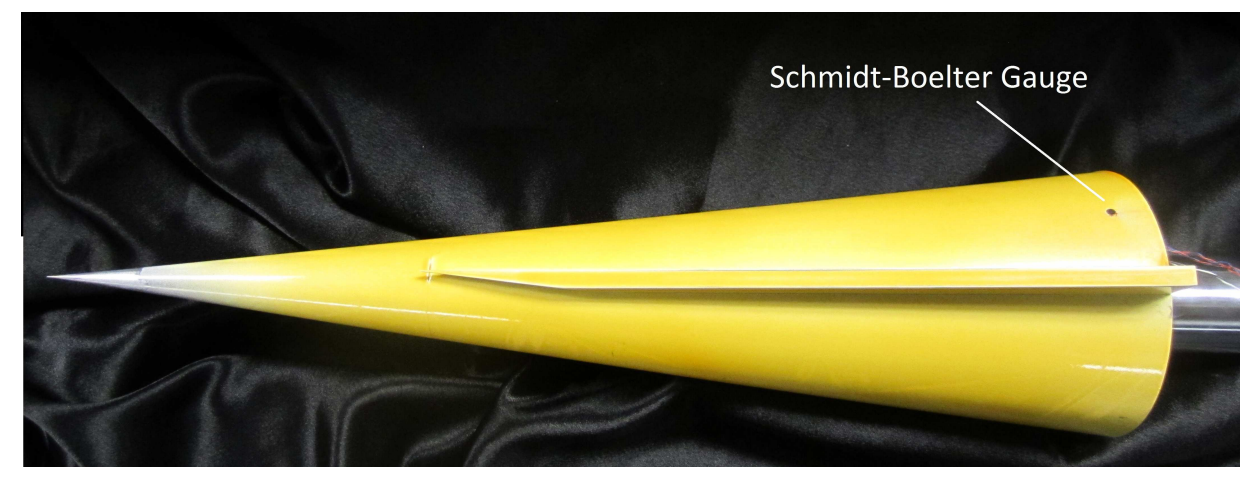

Figure 14. Modified Straight Cone Model. 
Results seen under both noisy and quiet flow show evidence of the vortex pairs seen in literature. ${ }^{21,22}$ Under noisy flow vortex growth moves upstream when compared to quiet flow results at similar Reynolds numbers, as shown in Fig. 15. Additionally, there is a transition front that occurs downstream of the onset of vortex spreading under noisy flow, as well as an artifact along the bottom of the model is thought to be a shock from the nozzle wall hot-film used in the BAM6QT. As the Reynolds number is increased the transition front and vortex spreading move upstream along the frusta of the cone, as shown in Fig. 16. The temperature differences between images are an artifact of the heating of the model throughout the course of a day of testing. This results in a lower relative temperature change as a day progresses.

At unit Reynolds numbers below approximately $R e=7.70 \times 10^{6} / \mathrm{m}$ under quiet flow, the vortices along the model appear to remain laminar. The vortex spreading in quiet flow begin to move forward as the Reynolds number increases. Figure 17 shows a detailed view of the TSP images for five different runs under quiet flow. The dark spot towards the rear of each image is the Schmidt-Boelter gauge. Interesting features are labeled in the first figure in which they appear. In 17(a), the lowest Reynolds number, the vortex appears to remain laminar along the entire body of the cone. At unit Reynolds numbers above approximately $\operatorname{Re}=8.38 \times 10^{6} / \mathrm{m}$ the vortices begin to spread, likely due to transition(Fig 17(b)). As the Reynolds number increases further in $17(\mathrm{c}), 17(\mathrm{~d})$, and $17(\mathrm{e})$, the spreading front moves further upstream. A secondary feature, likely the secondary vortex predicted by Korkegi and seen by Hummel ${ }^{25-27}$ can be seen below the original vortex, and also moves upstream as the Reynolds number increases. The vortex spreading seen here is a strong indicator of transition, however transition cannot be inferred with absolute certainty without additional instrumentation.

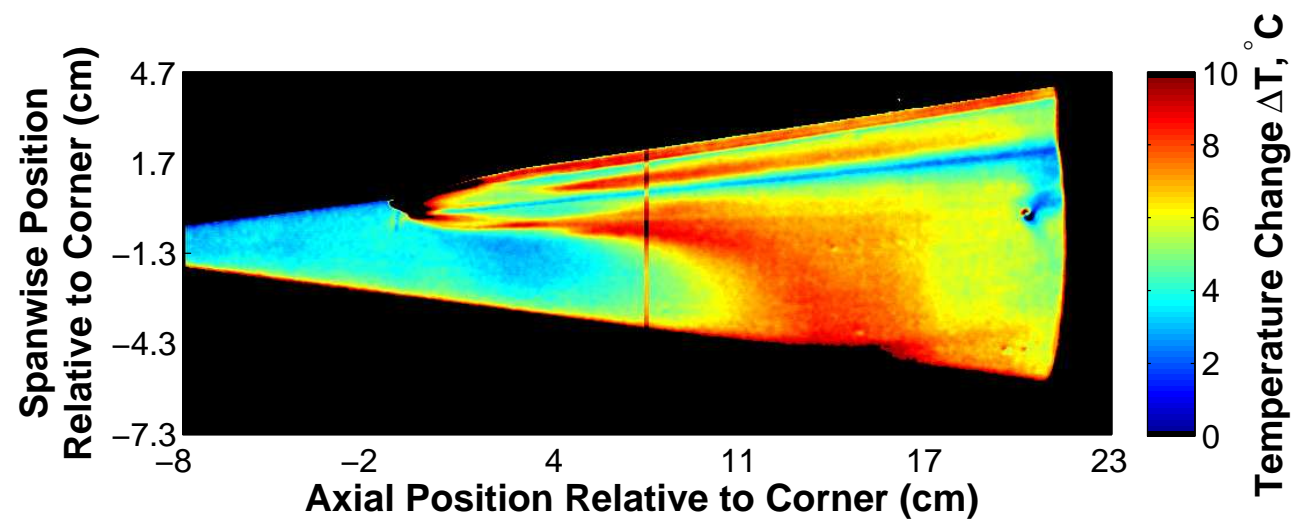

(a) Tunnel Conditions $\operatorname{Re}=11.83 \times 10^{6} / \mathrm{m} P_{o}=149$ psia Noisy Flow

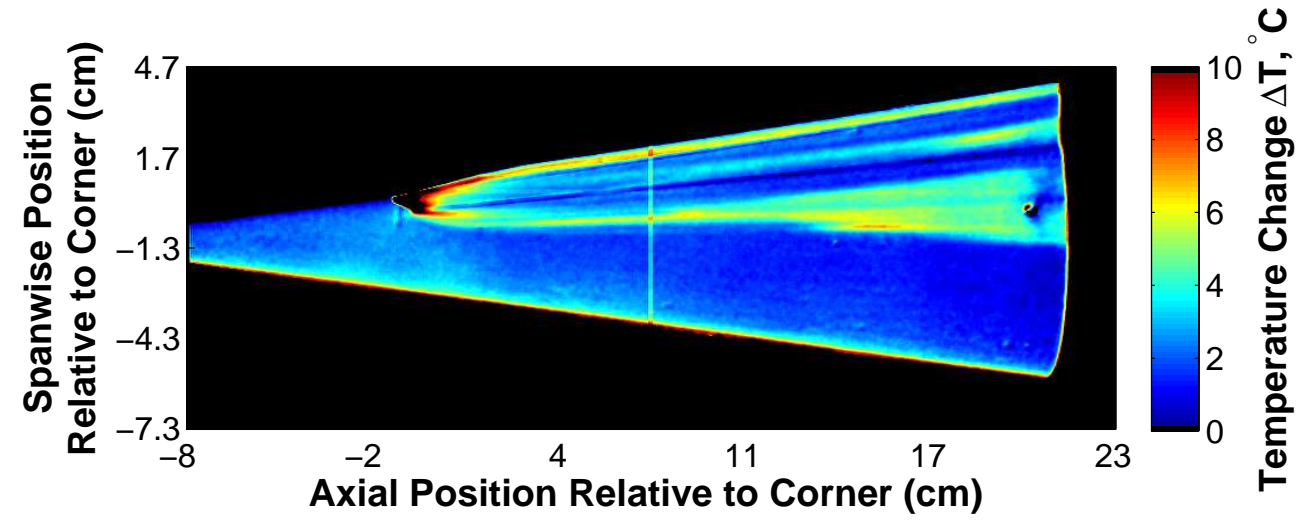

(b) Tunnel Conditions $\operatorname{Re}=10.94 \times 10^{6} / \mathrm{m} P_{o}=149$ psia Quiet Flow

Figure 15. Effect of tunnel noise on vortex spreading. 


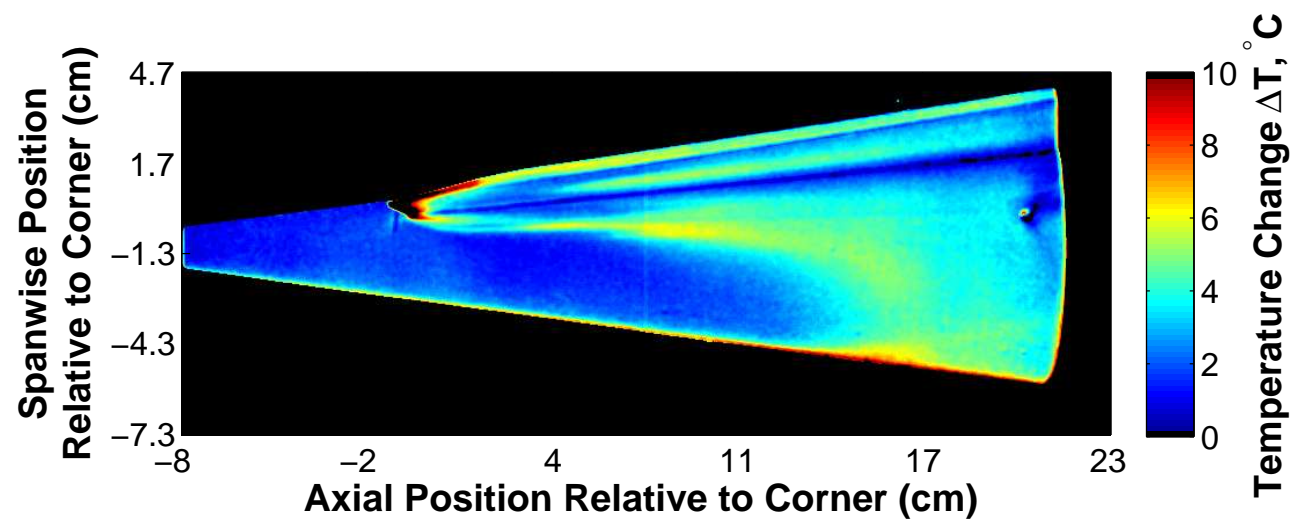

(a) Tunnel Conditions $\operatorname{Re}=9.05 \times 10^{6} / \mathrm{m} P_{o}=114$ psia

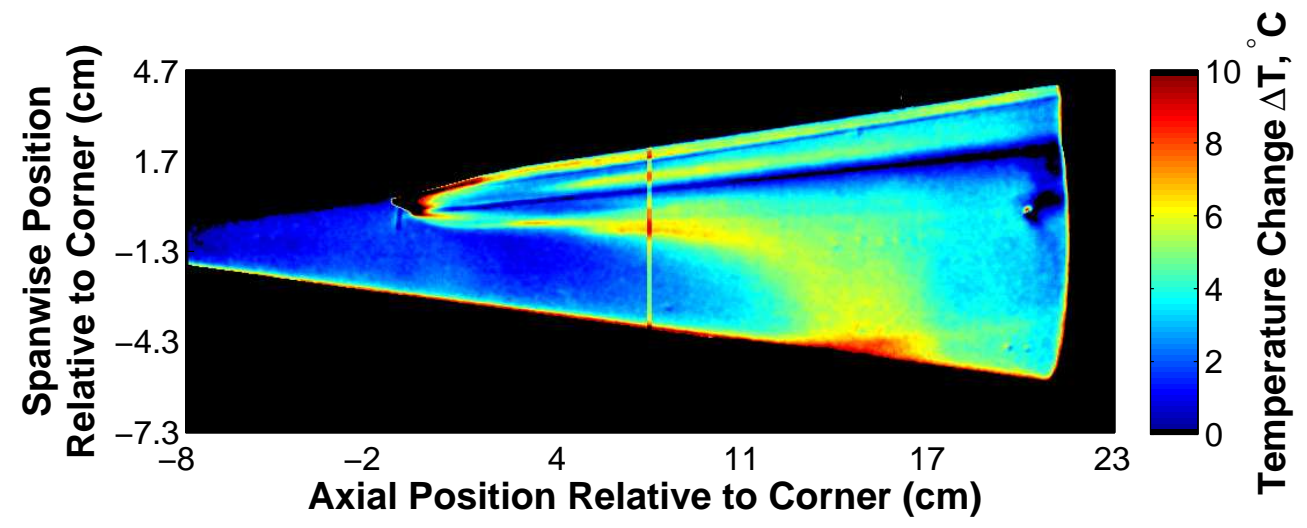

(b) Tunnel Conditions $\operatorname{Re}=10.81 \times 10^{6} / \mathrm{m} P_{o}=137$ psia

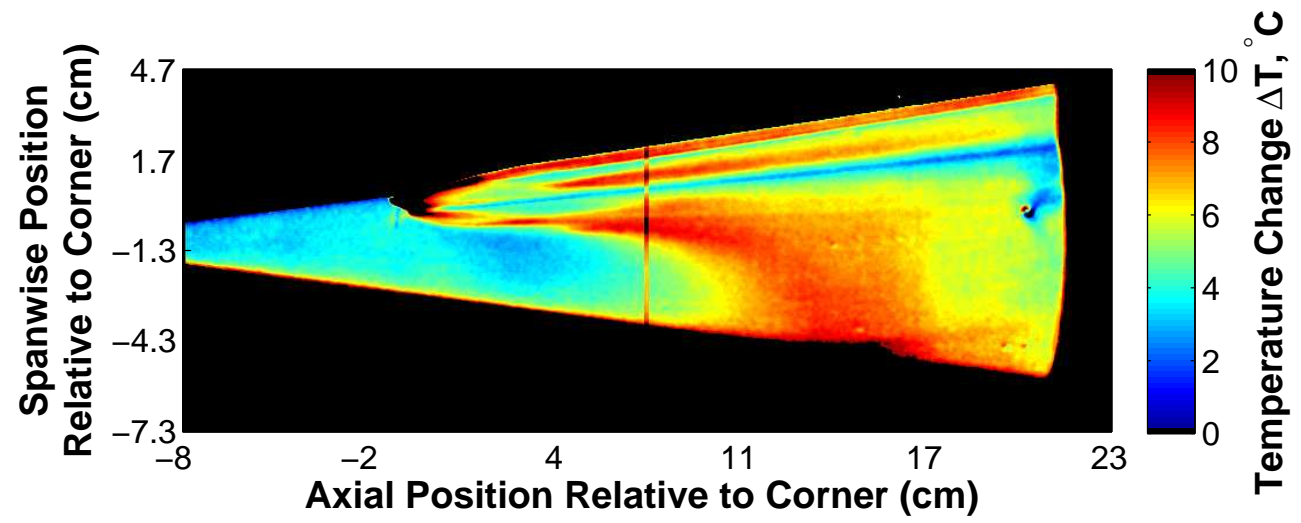

(c) Tunnel Conditions $\operatorname{Re}=11.83 \times 10^{6} / \mathrm{m} P_{o}=149$ psia

Figure 16. Transition front moves downstream with decreasing Reynolds number under noisy flow. 


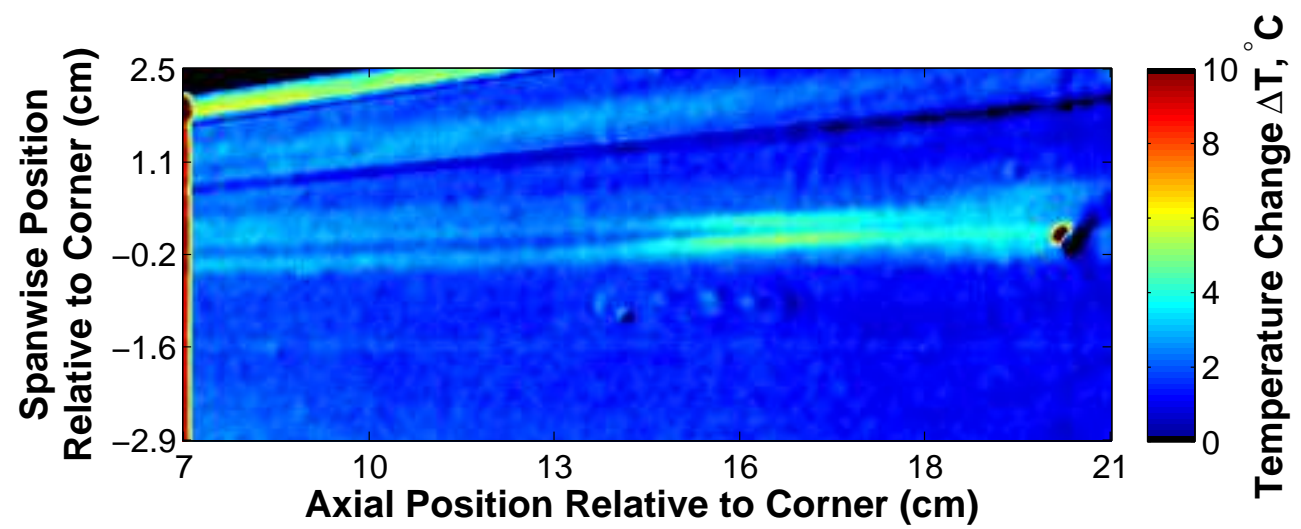

(a) [Tunnel Conditions $\operatorname{Re}=7.70 \times 10^{6} / \mathrm{m} P_{o}=105$ psia

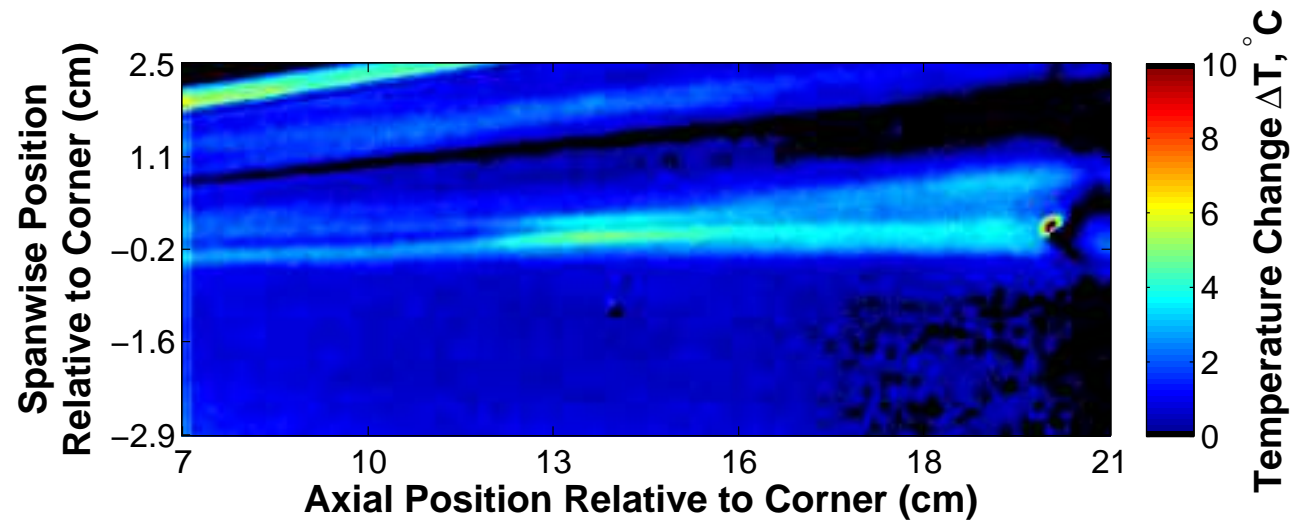

(b) Tunnel Conditions $\operatorname{Re}=8.38 \times 10^{6} / \mathrm{m} P_{o}=114$ psia

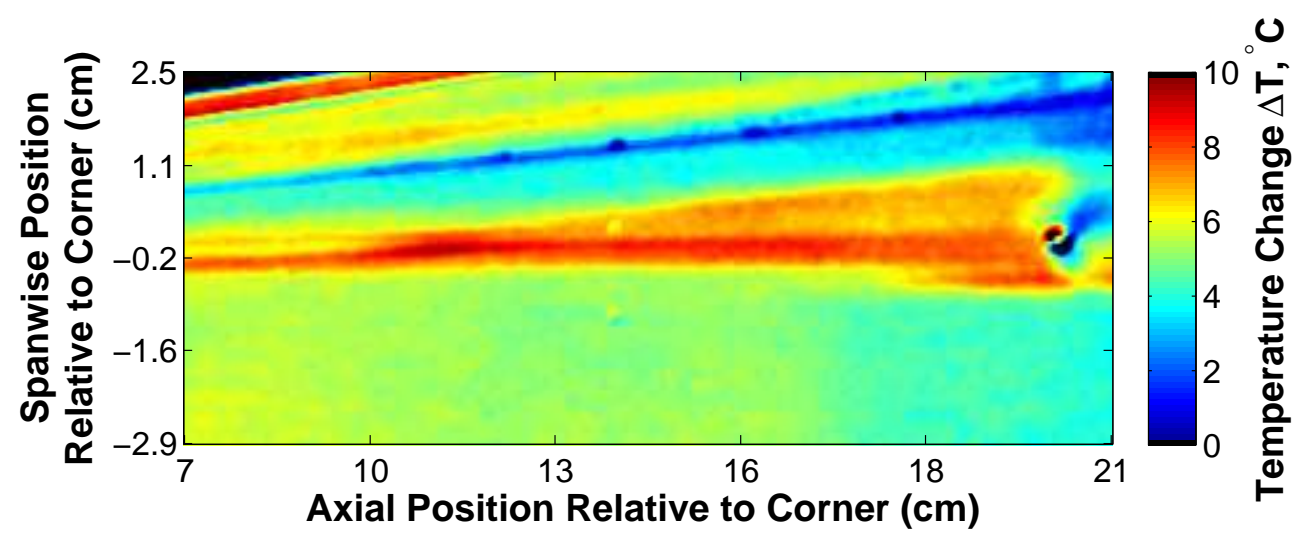

(c) Tunnel Conditions $\operatorname{Re}=9.25 \times 10^{6} / \mathrm{m} P_{o}=126$ psia

Figure 17. Vortex Spreading increases with increasing Reynolds number under quiet flow. 


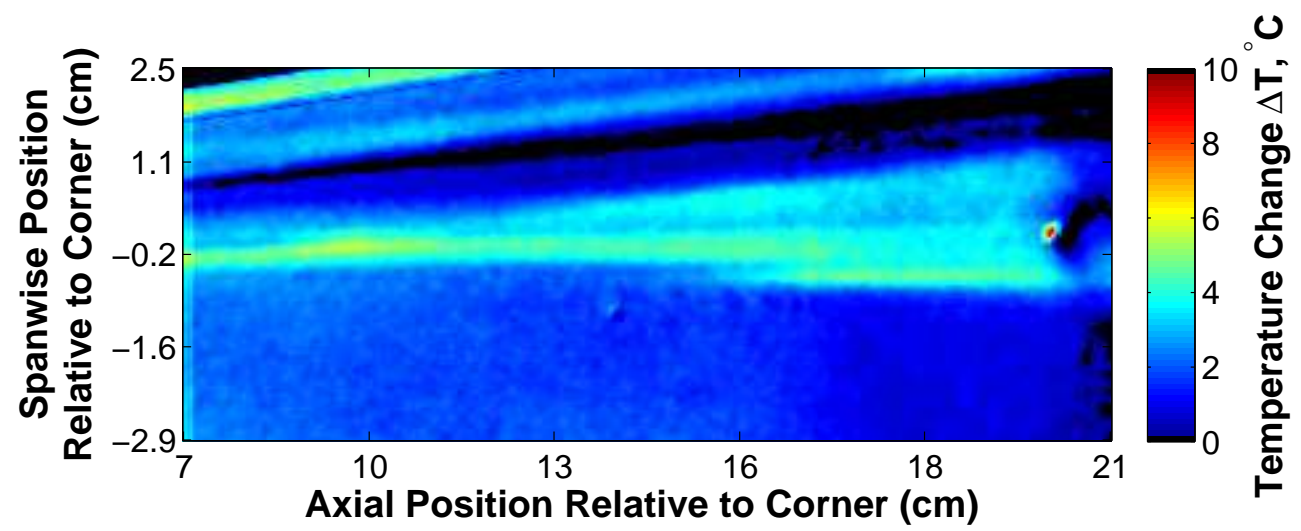

(d) Tunnel Conditions $\operatorname{Re}=9.91 \times 10^{6} / \mathrm{m} P_{o}=135$ psia

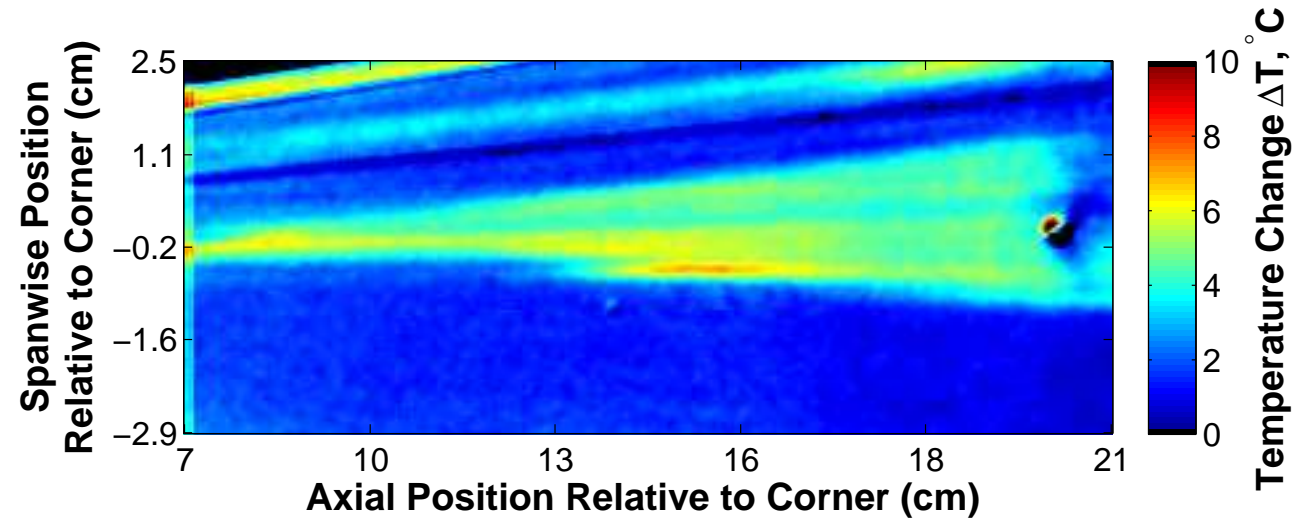

(e) Tunnel Conditions $\operatorname{Re}=10.94 \times 10^{6} / \mathrm{m} P_{o}=149$ psia

Figure 17. Vortex Spreading increases with increasing Reynolds number under quiet flow. (cont.) 


\section{Efforts Towards Calibration of PCB-132 Sensors and Construction of a Shock Tube}

Measurements of tunnel noise and boundary-layer instabilities have been uncommon in hypersonic tunnels, mostly due to the difficulty of performing such measurements. Boundary-layer instabilities on models in hypersonic tunnels consist of low-amplitude, high-frequency fluctuations. Few instruments that are sensitive enough to measure the instabilities are also robust enough to survive inside a hypersonic wind tunnel. Hot wires have been the usual method of measurement in the past, ${ }^{28-30}$ but there are several disadvantages to the use of these sensors. While hot wires are capable of surviving in some hypersonic wind tunnels, their strength is marginal and they are prone to breaking. In many of the larger production tunnels where flight vehicles are tested, the conditions are too harsh for hot wires to survive at all.

The finding that some high-frequency pressure transducers can be used to measure second-mode waves is, therefore, of clear interest. ${ }^{31}$ Second-mode waves, identified by Mack, ${ }^{32}$ are the dominant instability on flat plates and cones at zero angle of attack for Mach numbers above about 5 . They can also be important for cones at low angle of attack and nearly 2D or axisymmetric geometries, such as scramjet forebodies or re-entry vehicles.

Pressure transducers can be mounted flush with a model's surface, so that multiple sensors can be placed along a single streamline, reducing the number of runs required to measure the development of the instabilities. The PCB-132 model of pressure transducer (see Ref. 33) has also proven to be quite robust, capable of surviving in many hypersonic tunnels, with a low incidence of broken sensors. ${ }^{34-39}$ These qualities make attempting instability measurements feasible, even in many of the large tunnels used to test vehicles.

\section{A. PCB-132 Sensors}

PCB-132 sensors are piezoelectric pressure transducers designed to measure the time of arrival of shock waves. They are high-pass filtered at $11 \mathrm{kHz}$, with a quoted resonant frequency above $1 \mathrm{MHz}$. The manufacturer calibrates the sensors in a shock tube, by running one shock with a strength close to $7 \mathrm{kPa}$ past the sensor. The calibration is assumed to be linear, with a $0 \mathrm{~V}$ offset.

The manufacturer's calibration is not necessarily relevant or sufficiently accurate for the purposes of instability measurements. The response for an input of $7 \mathrm{kPa}$ is not necessarily similar to the response for an instability wave, which has pressure fluctuations three orders of magnitude smaller. In addition, the frequency response for the sensor is not identified. Second-mode instabilities in wind tunnels typically have frequencies between 100 and $600 \mathrm{kHz}$, so the frequency response of the sensor may be important to determining the actual magnitude of the pressure fluctuations across this frequency range.

Another issue with PCB-132 sensors is their spatial resolution. The instability waves on models generally have wavelengths on the order of millimeters. The sensing surface on the PCB sensors is 0.125 inches, or about $3.2 \mathrm{~mm}$, which is often longer than the second-mode wavelength. However, the sensing element is only a $0.03 \times 0.03$-in square $(0.762 \times 0.762 \mathrm{~mm})$. If the sensor size is significant compared to the second-mode wavelength, there will be spatial averaging. This averaging must be taken into account to find accurate amplitudes, so it is necessary to know over what area the sensor is measuring.

While the sizes of the sensing surface and sensing element are known, the area over which the sensor actually senses pressure (the active sensing area), is unknown. This is because the sensing surface and sensing element are both covered with a conductive epoxy. Pressure is transmitted to the sensing element through the epoxy, but the manner in which this happens is not well-defined. The sensing area may depend on the magnitude of the pressure fluctuation, as well as the actual thickness of the layer, which may vary between sensors. This makes it necessary to determine the sensing area of the sensors while calibrating them.

\section{B. Method of Calibration}

The most obvious method to calibrate the sensors is to create pressure fluctuations at fixed frequencies and known magnitudes and measure the sensor response. However, creating controlled fluctuations at the high frequencies required is very difficult. Ultrasonic emitters cannot readily reach the high end of the PCB frequency range, and accurate reference sensors to confirm the magnitude of the pressure fluctuations are difficult to find at these high frequencies.

An alternative is to use a step input or impulse as the calibration input. In theory, these inputs excite all response frequencies, enabling the entire frequency response to be identified using a single input. The 
high-frequency content is small so that averaging multiple step responses is likely required to obtain good high-frequency signal.

For a pressure transducer, a step input can be approximated with a shock wave. These can be generated using a shock tube or a laser perturber, but the flow in a shock tube is better understood. For this reason, it was decided to attempt to calibrate the PCB sensors using a shock tube. In order to calibrate for instability measurements, very weak, thin shock waves must be created. Thin shock waves are required to approximate the step input. Since a shock has some finite thickness, it is not really a step input, but if the shock passes over the sensor in a time sufficiently small compared to the response time of the sensor, it will closely approximate a step input. The rise time of PCB-132 sensors depends on the input voltage, varying from 65 to 312 nanoseconds for output voltages between $1 \mathrm{~V}$ and $5 \mathrm{~V}$ (about $70 \mathrm{kPA}$ and $340 \mathrm{kPa}$, respectively).

\section{Shock Strength and Passage Time}

Unfortunately, a small shock passage time and a weak shock are competing goals, since a shock becomes thicker and moves more slowly as it becomes weaker. This can be mitigated somewhat by using a low driven pressure in the shock tube, since the strength of the shock depends on the pressure ratio across the shock, and not the actual pressures. With a low driven pressure, the pressure ratio can be large even if the pressure difference across the shock is small. This can only work to a point, since eventually the driven section becomes rarefied and the shock begins to thicken again as the mean free path increases.

The time for the shock to pass over the sensor also depends heavily on the way in which the sensor is mounted. The two mounting configurations are static and pitot. The static configuration means that the sensor is mounted in the wall of the tube, so that it measures the rise in static pressure. The pitot configuration means that the sensor is mounted to point into the flow, so that it measures the rise in pitot pressure. In static configuration, the shock needs to travel over the entire sensing area before the input is complete. In pitot configuration, the shock encounters the entire sensing area at once, so the input is completed once the shock has completely passed through the plane of the sensor. Since the length of the sensing area is much larger than the thickness of the shock, the input takes much longer in static configuration, making it harder to create a step input.

The pitot pressure step is larger than the static pressure step, so again it becomes difficult to have both a small pressure rise and a short input duration. It may be necessary to identify the frequency response in pitot configuration using shocks too strong to be relevant to instability measurements, and then find the calibration curve of the sensors using shocks too thick to identify the frequency response. Assuming that the frequency response does not depend much on the magnitude of the input, this method should yield good calibrations. Calibration curves will be obtained in both configurations to see if the configuration affects the linearity or slope of the curve. Using thick shocks with sensors in pitot configuration should show how much the impulse duration affects the calibration.

\section{Existing Shock Tube Measurements}

Some experiments were performed in the existing shock tube at Purdue located in Armstrong Hall. This shock tube has an internal diameter of $10.8 \mathrm{~cm}$, a $0.64 \mathrm{~m}$ driver section, and a $4.67 \mathrm{~m}$ driven section. Weak shocks could not be created in this shock tube because of the poor vacuum performance of the tube, which prevented reaching low driven pressures. However, the sensors could be calibrated over a reasonable range of pressures when mounted in static configuration.

The pressure rise across the shock was calculated from the ideal shock equations based on the speed of the shock and measured by the reference pressure sensors (Kistler 603B1 piezoelectric transducers). The speed of the shock was calculated from the arrival times at two different sensor locations. The measurements do not agree with the calculations, and it is unclear which is more accurate. Both are shown in Figure 17.

It is clear in Figure 17 that the calibrations are linear over the measured range for both sensors. The manufacturer's calibration falls between the the two calibrations found for each sensor, indicating that for this range of pressures, the manufacturer's calibration gives at least a good estimate of the pressure measured by the sensor.

The sensor shock response was measured in both static and pitot configurations, as shown in Figure 18. The two responses are generally similar, showing a sharp rise when the shock passes, followed by a slower decline back to $0 \mathrm{~V}$ after about $0.1 \mathrm{~ms}$. This is expected, since the sensors are high-pass filtered at $11 \mathrm{kHz}$. The decline takes slightly longer in static configuration, probably due to the longer impulse time. Note that 


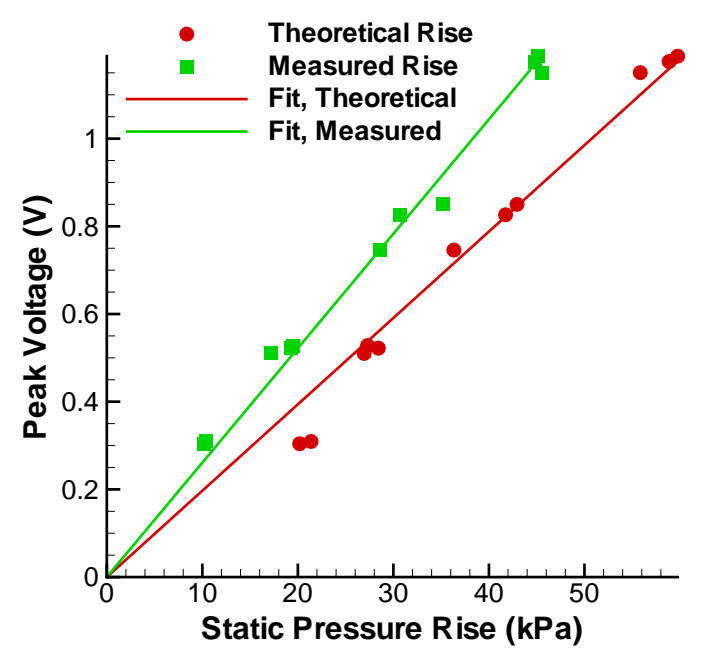

(f) Calibration for PCB \#5045

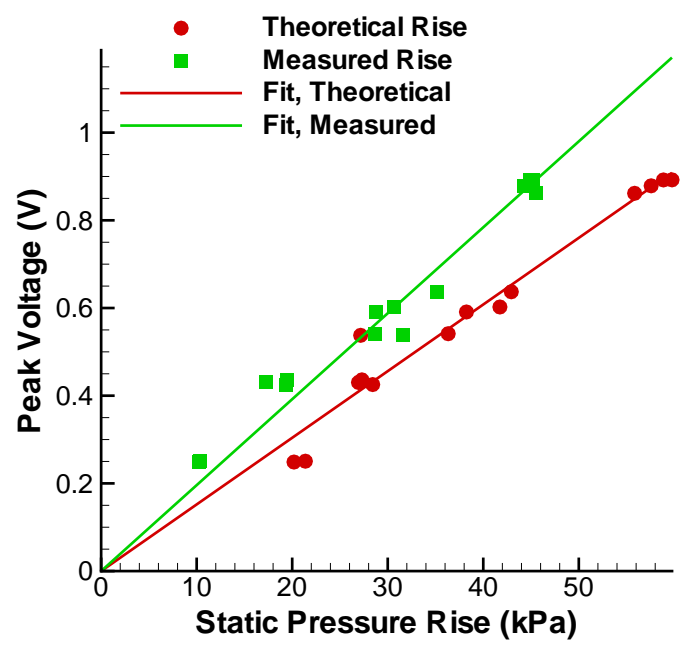

(g) Calibration for PCB \#5194

Figure 17. Calibration curves for two PCB-132A31 sensors.

in static configuration, the shock reaches the sensor at about $0.75 \mathrm{msec}$, not $0 \mathrm{msec}$. The major difference between the two is that a high-frequency oscillation is present in the pitot response. The frequency of this oscillation varies between sensors, and was observed to occur between $800 \mathrm{kHz}$ and $1.2 \mathrm{MHz}$. This oscillation is caused by excitation of the resonant frequency of the sensor. The resonance may not be excited in static configuration due to the longer impulse duration. In addition, in pitot configuration, the response to a given pressure rise is amplified by more than $300 \%$ compared to the response in static configuration. The reason for this amplification is uncertain. It is possible that the sensor responds differently in pitot configuration since all of the sensing area is excited at once, which may have a nonlinear effect on the response. The piezoelectric sensor may also be experiencing an acceleration effect, since these sensors are not compensated for acceleration.

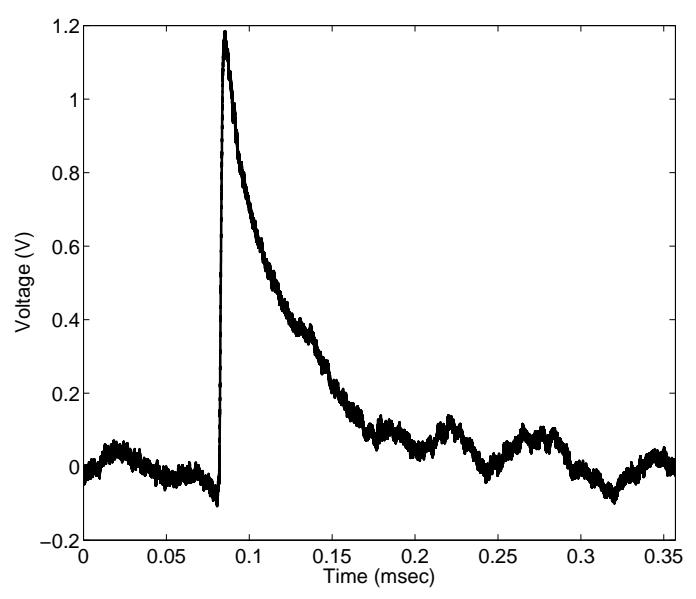

(a) Static configuration

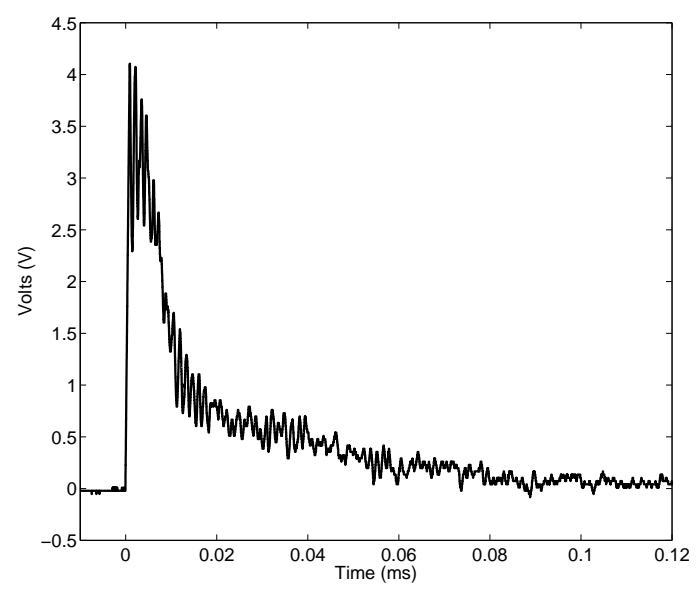

(b) Pitot configuration

Figure 18. PCB shock responses. 


\section{E. New Shock Tube}

In order to create the weak shocks required for calibration, a new shock tube has been built with a design based on the 6-inch shock tube in the Graduate Aerospace Laboratories at Caltech (GALCIT). ${ }^{40}$ The new shock tube (Figure 19) has a 3.5-inch $(15.2-\mathrm{cm})$ inner diameter, a 12-foot $(3.6-\mathrm{m})$ driven section, and a fourfoot $(1.2-\mathrm{m})$ driver section. PCB has expressed interest in the data that may be gathered from this shock tube, and will be cooperating with the development of the calibration techniques. They have also expressed interest in developing a new sensor designed for instability measurements and testing it in this shock tube.

The performance of this shock tube is currently being characterized. The driven section should be able to reach pressures of 1 millitorr (100 Pa) using an Oerlikon TRIVAC D4B vacuum pump, and the driver section is designed to withstand pressures as high as $6895 \mathrm{kPa}$. At this time, the minimum pressure reached in the driven tube has been 1.4 millitorr, which is sufficient for most purposes. The current maximum pressure is $970 \mathrm{kPa}$, which is the supply pressure in the building. The interior of the tube was honed, and the joints of the shock tube have been designed to be smooth, so as to avoid disturbing the flow and create a clean planar shock wave with a following laminar boundary layer. A laser-differential interferometer (LDI) may be used as a reference sensor to measure the thickness of the shock waves that pass. This shock tube will enable the measurement of weak waves, to check the calibration of the sensors to low amplitudes. The current estimate of the smallest static pressure rise achievable in this shock tube is $7 \mathrm{~Pa}$, which is within the upper range of second-mode amplitudes in wind tunnels. It should also be possible to perform repeated low-noise measurements, so that the frequency response of the sensors can be identified.

In order to use weak diaphragms at low driven pressures, it is necessary to reduce the driver section to pressures around $7 \mathrm{kPa}$. To allow for this, the driver section is connected to the vacuum system close to the end of the driven section. Cut-off valves allow the driven section to continue to be pumped down after the driver section has reached the appropriate pressure and protect the vacuum system from the high pressures that will sometimes be present in the driver section.

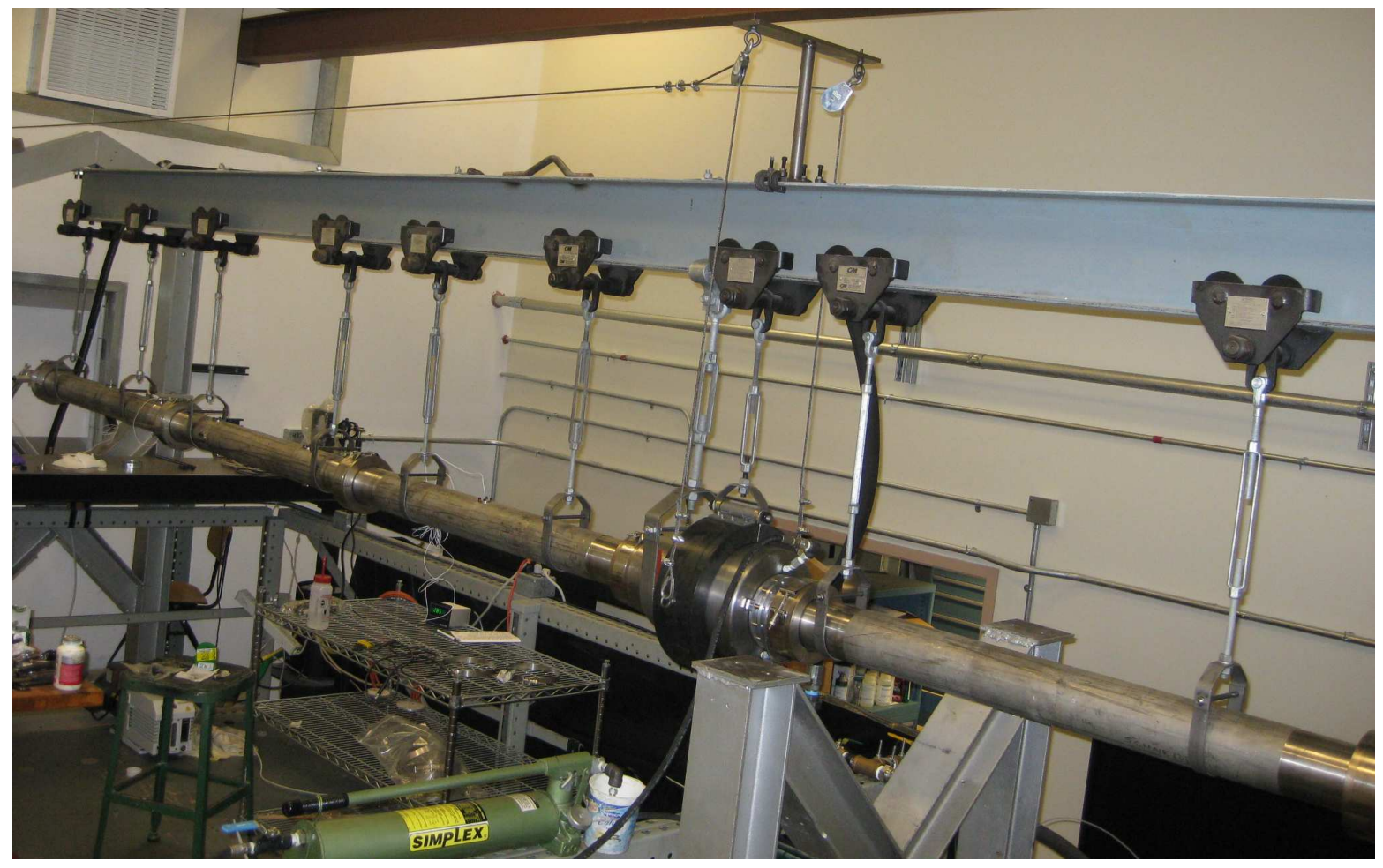

Figure 19. The new shock tube at Purdue. 


\section{Conclusions}

A 7-deg half angle cone at 6-deg angle of attack with temperature-sensitive paint and PCB pressure transducers was tested in the BAM6QT under quiet flow. On the lee side of the cone, the crossflow vortices were visualized with the TSP, and appear to break down to turbulence for sufficiently high Reynolds numbers. PCB gauges installed near the downstream end of the cone also appear to show turbulent or transitional flow. A DNS simulation qualitatively matches the observations made in the experiments, showing the stationary vortices beginning to break down near the lee ray. The stationary vortices visible on the yaw side of the cone did not begin to break down to turbulence, and the PCB gauges were unable to measure the travelling waves.

Attempts were made to use distributed roughness elements made from epoxy dots to control the spacing of hot streaks observed during transition on the flared cone under quiet flow. The elements used were apparently too large, causing horseshoe vortices visible in the TSP and damping the second-mode waves. Smaller dots cannot currently be created very repeatably, but the use of a lower-viscosity epoxy to create the dots may fix this problem. When the dots were spaced widely, hot streaks could be observed forming between the roughness elements. Measurements with PCB-132 sensors indicated that the peak frequency of the second-mode waves between the dots had been reduced slightly when compared to the case with no dots, but the amplitude of the waves was largely unchanged.

Measurements were performed on a forward-facing cavity model as a means of testing a laser perturber in the BAM6QT. The laser perturbations were found to excite the cavity resonance, indicating that the perturber does work in this tunnel. It was found that the model needed to be close to the point where the perturbation was generated in order to detect the perturbation. It is not clear why this is the case, though the perturbation may not be created on-axis with the model, causing it to miss the model at larger distances. The minimum density at which perturbations could be formed in the tunnel was found to be $0.0107 \mathrm{~kg} / \mathrm{m}^{3}$, about $60 \%$ of the minimum density value in the test cell. The reason for the difference is unknown.

Experiments were performed on a modified $7^{\circ}$ half-angle straight cone to investigate corner flow transition at hypersonic velocities. Vortex spreading is observed in most cases under both noisy and quiet flow given a sufficiently high Reynolds number. Although the current data shows strong indications of transition, further measurements are required to determine with certainty.

A shock tube has recently been completed at Purdue which is designed for sensor calibrations. The driven section has been shown to reach pressures of 1.4 millitorr, and should be able to reach 1 millitorr. This vacuum performance is necessary to create very weak shock waves comparable to the second-mode waves found in wind tunnels. No calibration work has yet been performed in this shock tube, which is still being characterized. However, some work was done in another shock tube at Purdue. The PCB-132 sensors were observed to have a linear calibration curve over the middle of their measurement range. Their shock response was observed as well, and the resonant frequencies of the sensors were found to vary between 800 $\mathrm{kHz}$ and $1.2 \mathrm{MHz}$.

\section{Acknowledgments}

Jerry Hahn in the Purdue AAE department machine shop made the parts for the shock tube. Ana Kerlo, a graduate student at Purdue, also assisted with the design of the shock tube. Joe Jewell at Caltech sent drawings of the original shock tube. Jason Damazo, also at Caltech, provided information about the design and operation of the original shock tube. Professor Steven H. Collicott at Purdue designed the optics used in the laser perturber system. This work has been funded in part by AFOSR, NDSEG, and the NASA Aeronautics Scholarship Program.

\section{References}

\footnotetext{
${ }^{1}$ Schneider, S. P., "Hypersonic Laminar-Turbulent Transition on Circular Cones and Scramjet Forebodies," Progress in Aerospace Sciences, Vol. 40, No. 1-2, February 2004, pp. 1-50.

${ }^{2}$ Beckwith, I. and III, C. M., "Aerothermodynamics and Transition in High-Speed Wind Tunnels at NASA Langley," Annual Review of Fluid Mechanics, Vol. 22, 1990, pp. 419-439.

${ }^{3}$ Schneider, S. P., "Effects of High-Speed Tunnel Noise on Laminar-Turbulent Transition," Journal of Spacecraft and Rockets, Vol. 38, No. 3, 2001, pp. 323-333.

${ }^{4}$ Schneider, S. P., "Flight Data for Boundary-Layer Transition at Hypersonic and Supersonic Speeds," Journal of Spacecraft
} 
and Rockets, Vol. 36, No. 1, 1999, pp. 8-20.

${ }^{5}$ Hofferth, J., Bowersox, R., and Saric, W., "The Texas A\&M Mach 6 Quiet Tunnel: Quiet Flow Performance," AIAA Paper 2010-4794, June 2010.

${ }^{6}$ Saric, W. S., Reed, H. L., and White, E. B., "Stability and Transition of Three-Dimensional Boundary Layers," Annual Review of Fluid Mechanics, Vol. 35, 2003, pp. 413-440.

${ }^{7}$ Deyhle, H. and Bippes, H., "Disturbance Growth in an Unstable Three-Dimensional Boundary Layer and its Dependence on Environmental Conditions," Journal of Fluid Mechanics, Vol. 316, December 1996, pp. 73-113.

${ }^{8}$ Ward, C. A., Wheaton, B. M., Chou, A., Berridge, D. C., Letterman, L. E., Luersen, R. P., and Schneider, S. P., "Hypersonic Boundary-Layer Transition Experiments in the Boeing/AFOSR Mach-6 Quiet Tunnel," AIAA Paper 2012-0282, Jan 2012.

${ }^{9}$ Sullivan, J. P., Schneider, S. P., Liu, T., Rubal, J., Ward, C., Dussling, J., Rice, C., Foley, R., Cai, Z., Wang, B., and Woodiga, S., "Quantitative Global Heat Transfer in a Mach-6 Quiet Tunnel," Final NASA Technical Report for Cooperative Agreement NNX08AC97A, November 2011.

${ }^{10}$ Gronvall, J. E., Johnson, H., and Candler, G. V., "Hypersonic Three-Dimensional Boundary Layer Transition on a Cone at Angle of Attack," Tech. rep., AIAA Paper Number TBA, June 2012.

${ }^{11} \mathrm{Li}, \mathrm{F}$., Choudhari, M., Chang, C.-L., and White, J., "Analysis of Instabilities in Non-Axisymmetric Hypersonic Boundary Layers over Cones," AIAA Paper 2010-4643, June 2010.

12 "Medtherm Corporation, Uncertainty in Schmidt-Boelter Heat Tranfer Measurements," Personal Communication - Phone, May 2012.

${ }^{13}$ Luersen, R. P. K., Roughness Application Techniques for Manipulation of Second-Mode Waves on a Flared Cone at Mach 6 (tentative), Master's thesis, Purdue University, West Lafayette, IN, August 2012 (expected).

${ }^{14}$ Ladoon, D. W., Schneider, S. P., and Schmisseur, J. D., "Physics of Resonance in a Supersonic Forward-Facing Cavity," Journal of Spacecraft and Rockets, Vol. 35, No. 5, Sep-Oct 1998, pp. 626-632.

${ }^{15}$ Juliano, T. J., Segura, R., Borg, M. P., Casper, K. M., M. J. Hannon, J., Wheaton, B. M., and Schneider, S. P., "Starting Issues and Forward-Facing Cavity Resonance in a Hypersonic Quiet Tunnel," AIAA Paper 2008-3735, Jun 2008.

${ }^{16}$ Engblom, W. A., Goldstein, D. B., Ladoon, D. W., and Schneider, S. P., "Fluid Dynamics of Hypersonic Forward-Facing Cavity Flow," Journal of Spacecraft and Rockets, Vol. 34, No. 4, Jul-Aug 1997, pp. 437-444.

${ }^{17}$ Segura, R., Oscillations in a Forward-Facing Cavity Measured using Laser-Differential Interferometry in a Hypersonic Quiet Tunnel, Master's thesis, West Lafayette, IN, Dec 2007.

${ }^{18}$ Chou, A., Ward, C. A., Letterman, L. E., Luersen, R. P., Borg, M. P., and Schneider, S. P., "Transition Research with Temperature-Sensitive Paints in the Boeing/AFOSR Mach-6 Quiet Tunnel," AIAA Paper 2011-3872, Jun 2011.

${ }^{19}$ Schmisseur, J. D., Receptivity of the Boundary Layer on a Mach-4 Elliptic Cone to Laser-Generated Localized Freestream Perturbations, Ph.D. thesis, School of Aeronautics \& Astronautics, Purdue University, West Lafayette, IN, Dec 1997.

${ }^{20} \mathrm{Chou}$, A., Characterization of Laser-Generated Perturbations and Instability Measurements on a Flared Cone, Master's thesis, School of Aeronautics \& Astronautics, Purdue University, West Lafayette, IN, Dec 2010.

${ }^{21}$ Stainback, P. C., "An Experimental Investigation at a Mach number of 4.95 of Flow in the Vicinity of a $90^{\circ}$ Interior Corner Aligned With a Free-stream Velocity," Technical Note D-184, NASA, 1960.

${ }^{22}$ Stainback, P. C., "Heat-transfer Measurements at a Mach number of 8 in the Vicinity of a $90^{\circ}$ Interior Corner Aligned with a Free-stream Velocity," Technical Note D-2417, NASA, 1964.

${ }^{23}$ Korkegi, R. H., "Effect of Transition on Three-Dimensional Shock- Wave/Boundary-Layer Interaction," AIAA Journal, Vol. 10, March 1972, pp. 361-363.

${ }^{24}$ Matthew P. Borg, Steven P. Schneider, Thomas J. Juliano, "Effect of Freestream Noise on Roughness-Induced Transition for the X-51A Forebody," AIAA Paper 2008-0592, Purdue University, January 2008.

${ }^{25}$ R. H. Korkegi, "On the Structure of Three-Dimensional Shock-Induced Separated Flow Regions," A Collection of Papers in the Aerospace Sciences, Wright-Patterson AFB, 1976, AFAPL-TR-79-2126.

${ }^{26}$ D. Hummel, "Experimental Investigations on Blunt Bodies and Corner Configurations in Hypersonic Flow," AGARD Conference Procedings No. 428 Aerodynamics of Hypersonic Lifting Vehicles, AGARD, 1989.

${ }^{27}$ Hummel, D., "Axial Flow in Corners at Supersonic and Hypersonic Speeds," R-764, AGARD, 1990.

${ }^{28}$ Stetson, K. F. and Kimmel, R. L., "On Hypersonic Boundary-Layer Stability," Tech. rep., AIAA Paper 1992-0737, January 1992.

${ }^{29}$ Demetriades, A., "An experiment on the stability of hypersonic laminar boundary layers," Journal of Fluid Mechanics, Vol. 7, No. 3, 1960, pp. 385-396.

${ }^{30}$ Kendall, J., "Wind Tunnel Experiments Relating to Supersonic and Hypersonic Boundary-Layer Transition," AIAA Journal, Vol. 13, No. 3, 1975, pp. 290-299.

${ }^{31}$ Fujii, K., "Experiment of Two Dimensional Roughness Effect on Hypersonic Boundary-Layer Transition," Journal of Spacecraft and Rockets, Vol. 43, No. 4, July-August 2006, pp. 731-738.

${ }^{32}$ Mack, L. M., "Linear Stability Theory and the Problem of Supersonic Boundary-Layer Transition," AIAA Journal, Vol. 13, No. 3, 1975, pp. 278-289.

33 "PCB Piezotronics Model 132A31," http://pcb.com/spec_sheet.asp?model=132A31\\&item_id=5190.

${ }^{34}$ Estorf, M., Radespiel, R., Schneider, S. P., Johnson, H., and Hein, S., "Surface-Pressure Measurements of Second-Mode Instability in Quiet Hypersonic Flow," Tech. rep., AIAA Paper 2008-1153, January 2008.

${ }^{35}$ Casper, K. M., Beresh, S. J., Henfling, J. F., Spillers, R. W., Pruett, B., and Schneider, S. P., "Hypersonic Wind-Tunnel Measurements of Boundary-Layer Pressure Fluctuations," June 2009, revised November 2009.

${ }^{36}$ Alba, C. R., Casper, K. M., Beresh, S. J., and Schneider, S. P., "Comparison of experimentally measured and computed second-mode disturbances in hypersonic boundary-layers," January 2010. 
${ }^{37}$ Lukashevich, S., Maslov, A., Shiplyuk, A., Fedorov, A., and Soudakov, V., "Stabilization of high-speed bounday layer using porous coatings of various thicknesses," AIAA Paper 2010-4720, June-July 2010.

${ }^{38}$ Bounitch, A., Lewis, D. R., and Lafferty, J. F., "Improved Measurements of "Tunnel Noise" Pressure Fluctuations in the AEDC Hypervelocity Wind Tunnel No. 9," January 2011.

${ }^{39}$ Rufer, S. J. and Berridge, D. C., "Experimental Study of Second-Mode Instabilities on a 7-Degree Cone at Mach 6," June 2011.

${ }^{40}$ Smith, J. A., Coles, D., Roshko, A., and Prasad, A., "A Description of the GALCIT 6" Shock Tube," GALCIT Report FM-67-1, June 1967. 